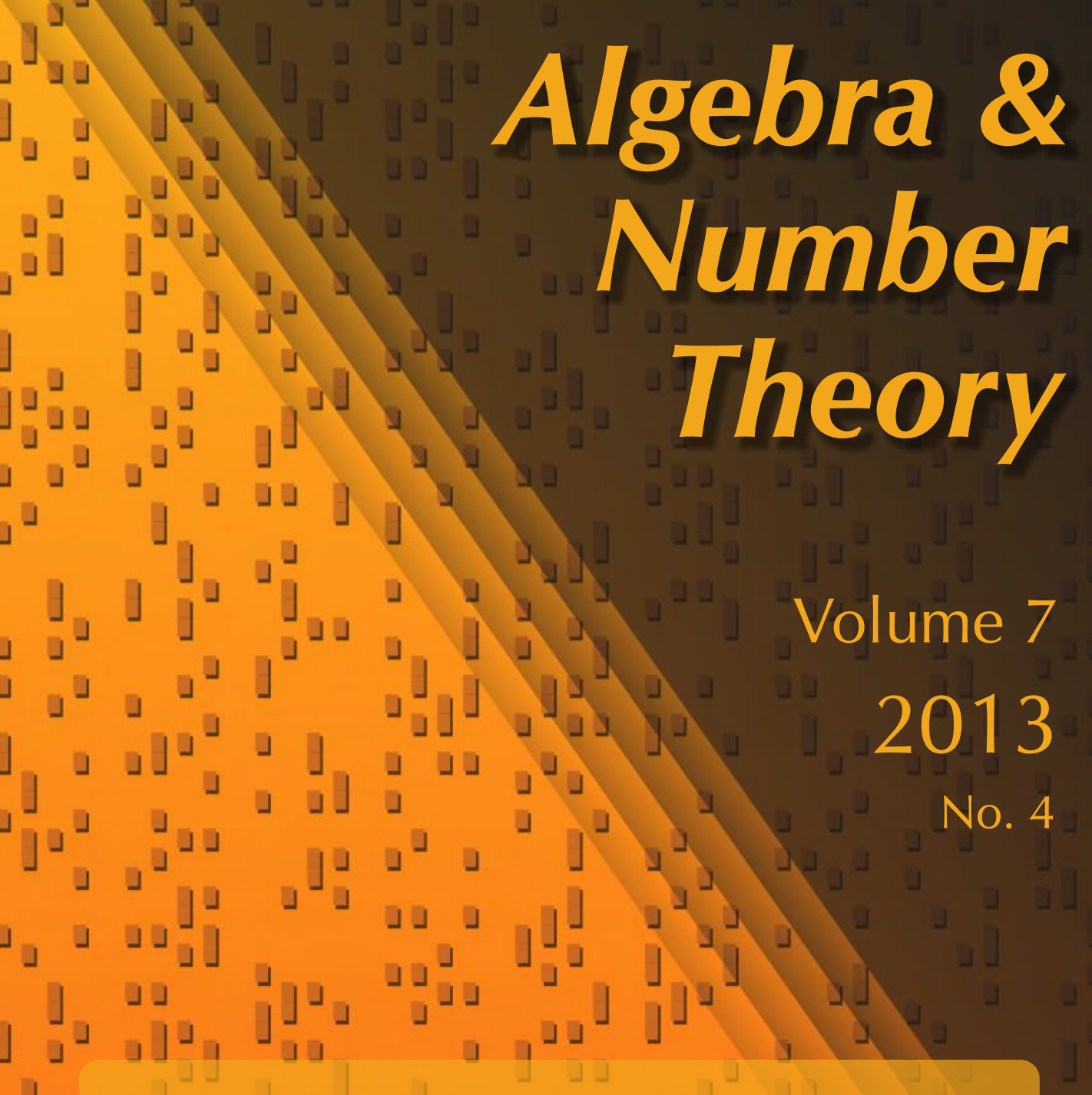

Adjoint ideals and a correspondence between log canonicity and $F$-purity

Shunsuke Takagi

\lrcorner

. 


\title{
Adjoint ideals and a correspondence between log canonicity and F-purity
}

\author{
Shunsuke Takagi
}

Dedicated to Professor Shihoko Ishii on the occasion of her sixtieth birthday

This paper presents three results on $F$-singularities. First, we give a new proof of Eisenstein's restriction theorem for adjoint ideal sheaves using the theory of $F$ singularities. Second, we show that a conjecture of Mustață and Srinivas implies a conjectural correspondence of $F$-purity and $\log$ canonicity. Finally, we prove this correspondence when the defining equations of the variety are very general.

\section{Introduction}

This paper deals with the theory of $F$-singularities, which are singularities defined using the Frobenius morphism in positive characteristic. We present three main results. First, we give a new proof of a restriction theorem for adjoint ideal sheaves. Second, we show that a certain arithmetic conjecture implies a conjectural correspondence of $F$-purity and $\log$ canonicity. Finally, we prove this correspondence when the defining equations of the variety are very general.

The notion of the adjoint ideal sheaf along a normal $\mathbb{Q}$-Gorenstein closed subvariety $X$ of a smooth complex variety $A$ with codimension $c$ was introduced in [Takagi 2010] (see Definition 1.8 for its definition). It is a modification of the multiplier ideal sheaf associated to the pair $(A, c X)$ and encodes much information on the singularities of $X$. Eisenstein [2010] recently proved a restriction theorem for these adjoint ideal sheaves. In this paper, we give a new proof of his result using the theory of $F$-singularities.

Building on earlier results [Hara and Yoshida 2003; Takagi 2004b; 2008], we introduced in [Takagi 2010] a positive characteristic analogue of the adjoint ideal sheaf called the test ideal sheaf (see Proposition-Definition 1.1). We conjectured that the adjoint ideal sheaf coincides after reduction to characteristic $p \gg 0$ with the test ideal sheaf and some partial results were obtained in [loc. cit.]. Making use of these results, we reduce the problem to an ideal theoretic problem on a normal

MSC2010: primary 13A35; secondary 14B05, 14F18.

Keywords: adjoint ideals, test ideals, $F$-pure singularities, log canonical singularities. 
$\mathbb{Q}$-Gorenstein ring essentially of finite type over a perfect field of characteristic $p>0$. The desired restriction formula is then obtained by adapting the argument of [Schwede 2009] (which can be traced back to [Fedder 1983]) to our setting (see Theorem 3.2). As a corollary, we show the correspondence between adjoint ideal sheaves and test ideal sheaves in a full generality (Corollary 3.4 on page 934).

The other ingredients of this paper are on a correspondence between $F$-pure singularities and log canonical singularities. $F$-pure singularities are defined via splitting of Frobenius morphisms (see Definition 1.3). Log canonical singularities form a class of singularities associated to the minimal model program (see Definition 1.7). It is known that the pair $(X ; t Z)$ is $\log$ canonical if its modulo $p$ reduction $\left(X_{p} ; t Z_{p}\right)$ is $F$-pure for infinitely many primes $p$, and the converse is conjectural (see Conjecture 2.4 for the precise statement). This conjecture is widely open, and only a few special cases are known. On the other hand, Mustață and Srinivas [2011, Conjecture 1.1] proposed the following more arithmetic conjecture to study a behavior of test ideal sheaves: if $V$ is a $d$-dimensional smooth projective variety over an algebraically closed field of characteristic zero, the action induced by the Frobenius morphism on the cohomology group $H^{d}\left(V_{p}, O_{V_{p}}\right)$ of its modulo $p$ reduction $V_{p}$ is bijective for infinitely many primes $p$. In this paper, we show that their conjecture implies the correspondence of $F$-purity and $\log$ canonicity (see Theorem 2.11). Our result can be viewed as strong evidence in favor of this conjectural correspondence although the conjecture of Mustață-Srinivas is also largely open.

As additional evidence of this correspondence, we consider the case when the defining equations of $X$ are very general. Shibuta and Takagi [2009] proved the correspondence if $X=\mathbb{C}^{n}$ and $Z$ is a complete intersection binomial subscheme or a space monomial curve. Using a similar idea, Hernández [2011] recently proved the case when $X=\mathbb{C}^{n}$ and $Z$ is a hypersurface of $X$ such that the coefficients of terms of its defining equation are algebraically independent over $\mathbb{Q}$. Using the techniques we have developed for Theorem 3.2, we generalize his result in Theorem 4.1 (page 935).

\section{Preliminaries}

Test ideals and $\boldsymbol{F}$-singularities of pairs. In this subsection, we briefly review the definitions of test ideal sheaves and $F$-singularities of pairs. The reader is referred to [Schwede 2008; 2009; 2011] and [Takagi 2004a; 2010] for the details.

Throughout this paper, all schemes are Noetherian, excellent and separated, and all sheaves are coherent. Let $A$ be an integral scheme of prime characteristic $p$. For each integer $e \geq 1$, we denote by $F^{e}: A \rightarrow A$ or $F^{e}: O_{A} \rightarrow F_{*}^{e} O_{A}$ the $e$-th iteration of the absolute Frobenius morphism on $A$. We say that $A$ is $F$-finite if $F: A \rightarrow A$ is a 
finite morphism. For example, every scheme essentially of finite type over a perfect field is $F$-finite. Given an ideal sheaf $I \subseteq 0_{A}$, for each $q=p^{e}$, we let $I^{[q]} \subseteq 0_{A}$ denote the ideal sheaf identified with $I \cdot F_{*}^{e} \mathscr{O}_{A}$ via the identification $F_{*}^{e} \mathscr{O}_{A} \cong O_{A}$. For a closed subscheme $Y$ of $A$, we let $\Phi_{Y}$ denote the defining ideal sheaf of $Y$ in $X$.

The notion of test ideal sheaves along arbitrary subvarieties was introduced in [Takagi 2010]. Below we give an alternate description of these sheaves based on the ideas of [Schwede 2009]. Let $A$ be a normal $\mathbb{Q}$-Gorenstein variety over an $F$-finite field of characteristic $p>0$ and $X \subseteq A$ be a reduced equidimensional closed subscheme of codimension $c$. Suppose that the Gorenstein index of $A$ is not divisible by $p$. There then exists infinitely many $e$ such that $\left(p^{e}-1\right) K_{A}$ is Cartier, and we fix such an integer $e_{0} \geq 1$. Grothendieck duality yields an isomorphism of $F_{*}^{e_{0}} \mathrm{O}_{A}$-modules

$$
F_{*}^{e_{0}} \mathscr{O}_{A} \cong \mathscr{H} \operatorname{om}_{\mathscr{O}_{A}}\left(F_{*}^{e_{0}}\left(\left(1-p^{e_{0}}\right) K_{A}\right), \mathscr{O}_{A}\right),
$$

and we let

$$
\varphi_{A, e_{0}}: F_{*}^{e_{0}} \mathrm{O}_{A}\left(\left(1-p^{e_{0}}\right) K_{A}\right) \rightarrow \mathfrak{O}_{A}
$$

denote the map corresponding to the global section 1 of $0_{A}$ via this isomorphism. When $A$ is Gorenstein, we can describe $\varphi_{A, e_{0}}$ more explicitly: it is obtained by tensoring the canonical dual $\left(F^{e_{0}}\right)^{\vee}: F_{*}^{e_{0}} \omega_{A} \rightarrow \omega_{A}$ of the $e_{0}$-times iterated Frobenius morphism $F^{e_{0}}: \mathrm{O}_{A} \rightarrow F_{*}^{e_{0}} \mathrm{O}_{A}$ with $\mathrm{O}_{A}\left(-K_{A}\right)$. Also, the composite map

$$
\varphi_{A, e_{0}} \circ F_{*}^{e_{0}} \varphi_{A, e_{0}} \circ \cdots \circ F_{*}^{(n-1) e_{0}} \varphi_{A, e_{0}}: F_{*}^{n e_{0}} \mathcal{O}_{A}\left(\left(1-p^{n e_{0}}\right) K_{A}\right) \rightarrow \mathcal{O}_{A}
$$

is denoted by $\varphi_{A, n e_{0}}$ for all integers $n \geq 1$. Just for convenience, $\varphi_{A, 0}$ is defined to be the identity map $\mathrm{O}_{A} \rightarrow \mathrm{O}_{A}$.

Proposition-Definition 1.1 (cf. [Takagi 2010, Definition 2.2]). Let the notation be as above, and let $Z:=\sum_{i=1}^{m} t_{i} Z_{i}$ be a formal combination, where the $t_{i}$ are nonnegative real numbers and the $Z_{i}$ are proper closed subschemes of $A$ that do not contain any component of $X$ in their support.

(1) There exists a unique smallest ideal sheaf $J \subseteq \mathcal{O}_{A}$ whose support does not contain any component of $X$ and that satisfies

$$
\varphi_{A, n e_{0}}\left(F_{*}^{n e_{0}}\left(J \mathscr{I}_{X}^{c\left(p^{n e_{0}}-1\right)} \oiint_{Z_{1}}^{\left\lceil t_{1}\left(p^{n e_{0}}-1\right)\right\rceil} \ldots \Im_{Z_{m}}^{\left\lceil t_{m}\left(p^{n e_{0}}-1\right)\right\rceil} \mathcal{O}_{A}\left(\left(1-p^{n e_{0}}\right) K_{A}\right)\right)\right) \subseteq J
$$

for all integers $n \geq 1$. This ideal sheaf is denoted by $\tilde{\tau}_{X}(A, Z)$. When $X=\varnothing$ (resp. $Z=\varnothing)$, we write simply $\tilde{\tau}(A ; Z)\left(\right.$ resp. $\left.\tilde{\tau}_{X}(A)\right)$.

(2) $(A, Z)$ is said to be purely $F$-regular along $X$ if $\tilde{\tau}_{X}(A, Z)=O_{A}$. 
Proof. We will prove that $\tilde{\tau}_{X}(A, Z)$ always exists. First, we suppose that $A$ is affine, $\mathrm{O}_{A}\left(\left(1-p^{n e_{0}}\right) K_{A}\right) \cong \mathfrak{O}_{A}$ and $\operatorname{Hom}_{\mathscr{C}_{A}}\left(F_{*}^{e_{0}} \mathcal{O}_{A}, \mathcal{O}_{A}\right)$ is generated by $\varphi_{A, e_{0}}$ as an $F_{*}^{e_{0}} \mathcal{O}_{A^{-}}$ module. Then $\operatorname{Hom}_{\mathscr{O}_{A}}\left(F_{*}^{n e_{0}} \mathcal{O}_{A}, \mathcal{O}_{A}\right)$ is generated by $\varphi_{A, n e_{0}}$ as an $F_{*}^{n e_{0}} \mathcal{O}_{A}$-module for all $n \geq 1$. Here we use the following fact:

Claim. There exists an element $\gamma \in \mathrm{O}_{A}$ not contained in any minimal prime ideal of $\mathscr{I}_{X}$ and satisfying the following property: for every $\delta \in \mathcal{O}_{A}$ not contained in any minimal prime of $\Im_{X}$, there exists an integer $n \geq 1$ such that

$$
\gamma \in \varphi_{A, n e_{0}}\left(F_{*}^{n e_{0}}\left(\delta \Phi_{X}^{c\left(p^{n e} e_{0}-1\right)} g_{Z_{1}}^{\left\lceil t_{1}\left(p^{n e} 0-1\right)\right\rceil} \ldots \oiint_{Z_{m}}^{\left\lceil t_{m}\left(p^{n e} 0-1\right)\right\rceil}\right)\right) .
$$

Proof. Suppose that $g \in \bigcap_{i} \mathscr{I}_{Z_{i}}$ is an element not contained in any minimal prime of $\mathscr{I}_{X}$ such that $\left.D(g)\right|_{X} \subseteq X$ is regular. By [Takagi 2010, Example 2.6], $D(g)$ is purely $F$-regular along $\left.D(g)\right|_{X}$. It then follows from an argument similar to [Schwede 2011, Proposition 3.21] that some power of $g$ satisfies the condition of the claim.

Let $\gamma \in \mathbb{O}_{A}$ be an element satisfying the conditions of the above claim. Then we will show that

$$
\tilde{\tau}_{X}(A, Z)=\sum_{n \geq 0} \varphi_{A, n e_{0}}\left(F_{*}^{n e_{0}}\left(\gamma \mathscr{I}_{X}^{c\left(p^{n e_{0}}-1\right)} \Phi_{Z_{1}}^{\left\lceil t_{1}\left(p^{n e_{0}}-1\right)\right\rceil} \ldots \mathscr{I}_{Z_{m}}^{\left\lceil t_{m}\left(p^{n e_{0}}-1\right)\right\rceil}\right)\right) .
$$

It is easy to check that $\sum_{n \geq 0} \varphi_{A, n e_{0}}\left(F_{*}^{n e_{0}}\left(\gamma \mathscr{G}_{X}^{c\left(p^{n e_{0}}-1\right)} g_{Z_{1}}^{\left\lceil t_{1}\left(p^{n e_{0}}-1\right)\right\rceil} \ldots \mathscr{I}_{Z_{m}}^{\left\lceil t_{m}\left(p^{n e_{0}}-1\right)\right\rceil}\right)\right)$ is the smallest ideal $J \subseteq \mathbb{O}_{A}$ containing $\gamma$ and satisfying

$$
\varphi_{A, n e_{0}}\left(F_{*}^{n e_{0}}\left(J \mathcal{F}_{X}^{c\left(p^{n e_{0}}-1\right)} \oiint_{Z_{1}}^{\left\lceil t_{1}\left(p^{n e_{0}}-1\right)\right\rceil} \ldots \mathscr{I}_{Z_{m}}^{\left\lceil t_{m}\left(p^{n e_{0}}-1\right)\right\rceil}\right)\right) \subseteq J
$$

for all $n \geq 1$. On the other hand, if an ideal $I \subseteq O_{A}$ is not contained in any minimal prime of $\mathscr{I}_{X}$ and satisfying

$$
\varphi_{A, n e_{0}}\left(F_{*}^{n e_{0}}\left(I \Im_{X}^{c\left(p^{n e_{0}}-1\right)} \oiint_{Z_{1}}^{\left\lceil t_{1}\left(p^{n e_{0}}-1\right)\right\rceil} \ldots \mathscr{\Phi}_{Z_{m}}^{\left\lceil t_{m}\left(p^{n e_{0}}-1\right)\right\rceil}\right)\right) \subseteq I
$$

for all $n \geq 1$, then $\gamma$ is forced to be in $I$ by definition. This completes the proof when $A$ is affine and $\operatorname{Hom}_{\mathscr{O}_{A}}\left(F_{*}^{e_{0}} \mathrm{O}_{A}, O_{A}\right)$ is generated by $\varphi_{A, e_{0}}$ as an $F_{*}^{e_{0}} \mathcal{O}_{A}$-module.

In the general case, $\tilde{\tau}_{X}(A, Z)$ is obtained by gluing the constructions on affine charts.

Remark 1.2. The definition of $\tilde{\tau}_{X}(A, Z)$ is independent of the choice of $e_{0}$.

Next, we will give a definition of $F$-singularities of pairs and $F$-pure thresholds.

Definition 1.3 ([Takagi 2006, Definition 3.1; Schwede 2008, Proposition 3.3], cf. [Schwede 2008, Proposition 5.3]). Let $X$ be an $F$-finite integral normal scheme of characteristic $p>0$ and $D$ be an effective $\mathbb{Q}$-divisor on $X$. Let $Z=\sum_{i=1}^{m} t_{i} Z_{i}$ be a formal combination, where the $t_{i}$ are nonnegative real numbers and the $Z_{i}$ are proper closed subschemes of $X$. Fix an arbitrary point $x \in X$. 
(i) $((X, D) ; Z)$ is said to be strongly $F$-regular at $x$ if, for every nonzero $\gamma \in \mathbb{O}_{X, x}$, there exist an integer $e \geq 1$ and $\delta \in \Phi_{Z_{1}, x}^{\left\lceil t_{1}\left(p^{e}-1\right)\right\rceil} \ldots \Phi_{Z_{m}, x}^{\left\lceil t_{m}\left(p^{e}-1\right)\right\rceil}$ such that

$$
\gamma \delta F^{e}: \mathscr{O}_{X, x} \rightarrow F_{*}^{e} \mathscr{O}_{X}\left(\left\lceil\left(p^{e}-1\right) D\right\rceil\right)_{x}, \quad a \mapsto \gamma \delta a^{p^{e}},
$$

splits as an $\mathbb{O}_{X, x}$-module homomorphism.

(ii) $((X, D) ; Z)$ is said to be sharply $F$-pure at $x$ if there exist an integer $e \geq 1$ and $\delta \in \Phi_{Z_{1}, x}^{\left\lceil t_{1}\left(p^{e}-1\right)\right\rceil} \ldots \Phi_{Z_{m}, x}^{\left\lceil t_{m}\left(p^{e}-1\right)\right\rceil}$ such that

$$
\delta F^{e}: \mathscr{O}_{X, x} \rightarrow F_{*}^{e} \mathcal{O}_{X}\left(\left\lceil\left(p^{e}-1\right) D\right\rceil\right)_{x}, \quad a \mapsto \delta a^{p^{e}},
$$

splits as an $\mathbb{O}_{X, x}$-module homomorphism.

We simply say that $(X ; Z)$ is strongly $F$-regular (resp. sharply $F$-pure) at $x$ if $((X, 0) ; Z)$ is. We say that $(X, D)$ is strongly $F$-regular (resp. sharply $F$-pure) if $((X, D) ; \varnothing)$ is. Also, we say that $((X, D) ; Z)$ is strongly $F$-regular (resp. sharply $F$-pure) if it is for all $x \in X$.

(iii) Suppose that $(X, D)$ is sharply $F$-pure at $x$. Then the $F$-pure threshold $\operatorname{fpt}_{x}((X, D) ; Z)$ of $Z$ at $x$ is defined to be $\operatorname{fpt}_{x}((X, D) ; Z):=\sup \left\{t \in \mathbb{R}_{\geq 0} \mid((X, D) ; t Z)\right.$ is sharply $F$-pure at $\left.x\right\}$.

We write simply $\operatorname{fpt}_{x}(X ; Z)$ when $D=0$.

Remark 1.4. Let $A$ and $Z$ be as in Proposition-Definition 1.1. Then $(A ; Z)$ is strongly $F$-regular at a point $x \in A$ if and only if $\tilde{\tau}(A, Z)_{x}=\mathcal{O}_{A, x}$.

There exists a criterion for sharp $F$-purity called the Fedder type criterion, which we will use later.

Lemma 1.5 [Fedder 1983, Lemma 1.6; Schwede 2008, Theorem 4.1]. Let A be an $F$-finite regular integral affine scheme of characteristic $p>0$ and $X \subseteq A$ be a reduced equidimensional closed subscheme.

(1) For each nonnegative integer e, the natural morphism

$$
F_{*}^{e}\left(g_{X}^{\left[p^{e}\right]}: \mathscr{I}_{X}\right) \cdot \mathscr{H} \mathrm{om}_{\mathscr{O}_{A}}\left(F_{*}^{e} \mathscr{O}_{A}, \mathscr{O}_{A}\right) \rightarrow \mathscr{H} \mathrm{om}_{\mathbb{O}_{X}}\left(F_{*}^{e} \mathscr{O}_{X}, \mathscr{O}_{X}\right)
$$

sending $s \cdot \varphi_{A}$ to $\overline{\varphi_{A} \circ F_{*}^{e}(\times s)}$ induces the isomorphism



(2) Let $Z=\sum_{i=1}^{m} t_{i} Z_{i}$ be a formal combination, where the $t_{i}$ are nonnegative real numbers and the $Z_{i}$ are proper closed subschemes of $A$ that do not contain any component of $X$ in their support. Let $x \in X$ be an arbitrary point. Then the following conditions are equivalent:

(a) $\left(X ;\left.Z\right|_{X}\right)$ is sharply $F$-pure at $x$. 
(b) There exists an integer $e_{0} \geq 1$ such that

$$
\left(\mathscr{\Phi}_{X, x}^{\left[p^{e_{0}}\right]}: \mathscr{I}_{X, x}\right) \mathscr{\Phi}_{Z_{1}, x}^{\left[t_{1}\left(p^{e_{0}}-1\right)\right\rceil} \cdots \mathscr{I}_{Z_{m}, x}^{\left\lceil t_{m}\left(p^{e_{0}}-1\right)\right\rceil} \nsubseteq \mathfrak{m}_{A, x}^{\left[p^{e_{0}}\right]},
$$

which is equivalent to saying that

$$
\left.\left(\mathscr{I}_{X, x}^{\left[p^{n e} e_{0}\right]}: \mathscr{I}_{X, x}\right) \mathscr{I}_{Z_{1}, x}^{\left\lceil t_{1}\left(p^{n e_{0}}-1\right)\right\rceil} \cdots \mathscr{I}_{Z_{m}, x}^{\left\lceil t_{m}\left(p^{n e} 0-1\right)\right\rceil} \nsubseteq \mathfrak{m}_{A, x}^{\left[p^{n e} 0\right.}\right]
$$

for all integers $n \geq 1$. Here, $\mathfrak{m}_{A, x} \subseteq \mathcal{O}_{A, x}$ denotes the maximal ideal of $x$.

We remark that (2) is an easy consequence of (1) in Lemma 1.5.

Singularities of the minimal model program. In this subsection, we recall the definitions of adjoint ideal sheaves, multiplier ideal sheaves and singularities of pairs. The reader is referred to [Lazarsfeld 2004] for basic theory of multiplier ideal sheaves and to [Eisenstein 2010; Takagi 2010] for that of adjoint ideal sheaves.

Let $X$ be a normal variety over an algebraically closed field $K$ of characteristic 0 , and let $Z=\sum_{i} t_{i} Z_{i}$ be a formal combination, where the $t_{i}$ are nonnegative real numbers and the $Z_{i}$ are proper closed subschemes of $X$. A log resolution of the pair $(X, Z)$ is a proper birational morphism $\pi: \widetilde{X} \rightarrow X$ with $X$ a smooth variety such that all scheme theoretic inverse images $\pi^{-1}\left(Z_{i}\right)$ are divisors and in addition $\bigcup_{i} \operatorname{Supp} \pi^{-1}\left(Z_{i}\right) \cup \operatorname{Exc}(\pi)$ is a simple normal crossing divisor. The existence of $\log$ resolutions is guaranteed by the desingularization theorem of Hironaka [1964].

Definition 1.6. Let $X$ and $Z$ be as above, and let $D:=\sum_{k} d_{k} D_{k}$ be a boundary divisor on $X$, that is, $D$ is a $\mathbb{Q}$-divisor on $X$ with $0 \leq d_{k} \leq 1$ for all $k$. In addition, we assume that $K_{X}+D$ is $\mathbb{Q}$-Cartier and no component of $\lfloor D\rfloor$ is contained in the support of the $Z_{i}$. Fix a log resolution $\pi: \widetilde{X} \rightarrow X$ of $(X, D+Z)$ such that $\pi_{*}^{-1}\lfloor D\rfloor$ is smooth. Then the adjoint ideal sheaf $\operatorname{adj}_{D}(X, Z)$ of $(X, Z)$ along $D$ is defined to be

$$
\operatorname{adj}_{D}(X, Z):=\pi_{*} \mathrm{O}_{\tilde{X}}\left(\left\lceil K_{\widetilde{X}}-\pi^{*}\left(K_{X}+D\right)-\sum_{i} t_{i} \pi^{-1}\left(Z_{i}\right)\right\rceil+\pi_{*}^{-1}\lfloor D\rfloor\right) \subseteq \mathrm{O}_{X} .
$$

When $D=0$, we denote this ideal sheaf by $\mathscr{f}(X, Z)$ and call it the multiplier ideal sheaf associated to $(X, Z)$.

Definition 1.7. Let $X$ and $Z$ be as above, and let $D$ be a $\mathbb{Q}$-divisor on $X$ such that $K_{X}+D$ is $\mathbb{Q}$-Cartier. Fix a $\log$ resolution $\pi: \widetilde{X} \rightarrow X$ of $(X, D+Z)$, and then we can write

$$
K_{\tilde{X}}=\pi^{*}\left(K_{X}+D\right)+\sum_{i} t_{i} \pi^{-1}\left(Z_{i}\right)+\sum_{j} a_{j} E_{j},
$$

where the $a_{j}$ are real numbers and the $E_{j}$ are prime divisors on $\widetilde{X}$. Fix an arbitrary point $x \in X$.

(i) $((X, D) ; Z)$ is said to be $k l t$ at $x$ if $a_{j}>-1$ for all $j$ such that $x \in \pi\left(E_{j}\right)$. 
(ii) $((X, D) ; Z)$ is said to be $\log$ canonical at $x$ if $a_{j} \geq-1$ for all $j$ such that $x \in \pi\left(E_{j}\right)$.

When $X$ is $\mathbb{Q}$-Gorenstein and $D=0$, we simply say that $(X ; Z)$ is klt (resp. log canonical) at $x$ instead of saying that $((X, 0) ; Z)$ is klt (resp. log canonical) at $x$. When $Z=\varnothing$, we simply say that $(X, D)$ is klt (resp. $\log$ canonical) at $x$ instead of saying that $((X, D) ; \varnothing)$ is klt (resp. $\log$ canonical) at $x$. Also, we say that $((X, D) ; Z)$ is klt (resp. $\log$ canonical) if it is so for all $x \in X$.

(iii) Suppose that $(X, D)$ is $\log$ canonical at $x$. Then the log canonical threshold $\operatorname{lct}_{x}((X, D) ; Z)$ of $Z$ at $x$ is defined to be

$$
\operatorname{lct}_{x}((X, D) ; Z):=\sup \left\{t \in \mathbb{R}_{\geq 0} \mid((X, D) ; t Z) \text { is log canonical at } x\right\} .
$$

We simply denote this threshold $\operatorname{lct}_{x}(X ; Z)$ if $X$ is $\mathbb{Q}$-Gorenstein and $D=0$.

When the ambient variety is smooth, we can generalize the notion of adjoint ideal sheaves to the higher codimension case. Let $A$ be a smooth variety over an algebraically closed field of characteristic 0 and $X \subseteq A$ be a reduced equidimensional closed subscheme of codimension $c$.

Definition 1.8 ([Takagi 2010, Definition 1.6]; cf. [Eisenstein 2010, Definition 3.4]). Let the notation be as above. Let $Z=\sum_{i} t_{i} Z_{i}$ be a formal combination, where the $t_{i}$ are nonnegative real numbers and the $Z_{i}$ are proper closed subschemes of $A$ that do not contain any component of $X$ in their support.

(i) Let $f: A^{\prime} \rightarrow A$ be the blow-up of $A$ along $X$ and $E$ be the reduced exceptional divisor of $f$ that dominates $X$. Let $g: \widetilde{A} \rightarrow A^{\prime}$ be a log resolution of $\left(A^{\prime}, f^{-1}(X)+\sum_{i} f^{-1}\left(Z_{i}\right)\right)$ so that the strict transform $g_{*}^{-1} E$ is smooth, and set $\pi=f \circ g$. Then the adjoint ideal sheaf $\operatorname{adj}_{X}(A, Z)$ of the pair $(A, Z)$ along $X$ is defined to be

$$
\operatorname{adj}_{X}(A, Z):=\pi_{*} \mathcal{O}_{\widetilde{A}}\left(K_{\widetilde{A} / A}-c \pi^{-1}(X)-\left\lfloor\sum_{i} t_{i} \pi^{-1}\left(Z_{i}\right)\right\rfloor+g_{*}^{-1} E\right) .
$$

(ii) $(A ; Z)$ is said to be plt along $X$ if $\operatorname{adj}_{X}(A, Z)=\mathbb{O}_{A}$.

Remark 1.9. (1) Definitions $1.6,1.7$ and 1.8 are independent of the choice of a $\log$ resolution used to define them.

(2) Let $X$ and $Z$ be as in Definition 1.7, and assume that $X$ is $\mathbb{Q}$-Gorenstein. Then $(X ; Z)$ is klt at a point $x \in X$ if and only if $\mathscr{E}(X, Z)_{x}=\mathscr{O}_{X, x}$.

\section{Reduction from characteristic 0 to characteristic $p$}

In this section, we briefly review how to reduce things from characteristic 0 to characteristic $p>0$. Our main references are [Hochster and Huneke 1999, Chapter 2; Mustață and Srinivas 2011, Section 3.2]. 
Let $X$ be a scheme of finite type over a field $K$ of characteristic 0 and $Z=\sum_{i} t_{i} Z_{i}$ be a formal combination, where the $t_{i}$ are real numbers and the $Z_{i}$ are proper closed subschemes of $X$. Choosing a suitable finitely generated $\mathbb{Z}$-subalgebra $B$ of $K$, we can construct a scheme $X_{B}$ of finite type over $B$ and closed subschemes $Z_{i, B} \subsetneq X_{B}$ such that there exist isomorphisms

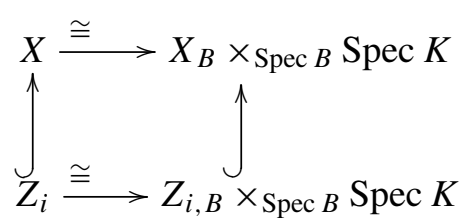

Note that we can enlarge $B$ by localizing at a single nonzero element and replacing $X_{B}$ and $Z_{i, B}$ with the corresponding open subschemes. Thus, applying the generic freeness [Hochster and Huneke 1999, (2.1.4)], we may assume that $X_{B}$ and the $Z_{i, B}$ are flat over $\operatorname{Spec} B$. Letting $Z_{B}:=\sum_{i} t_{i} Z_{i, B}$, we refer to $\left(X_{B}, Z_{B}\right)$ as a model of $(X, Z)$ over $B$. Given a closed point $\mu \in \operatorname{Spec} B$, we let $X_{\mu}$ (resp. $Z_{i, \mu}$ ) denote the fiber of $X_{B}$ (resp. $Z_{i, B}$ ) over $\mu$ and define $Z_{\mu}:=\sum_{i} t_{i} Z_{i, \mu}$. Then $X_{\mu}$ is a scheme of finite type over the residue field $\kappa(\mu)$ of $\mu$, which is a finite field of characteristic $p(\mu)$. If $X$ is regular, then after possibly enlarging $B$, we may assume that $X_{B}$ is regular. In particular, there exists a dense open subset $W \subseteq \operatorname{Spec} B$ such that $X_{\mu}$ is regular for all closed points $\mu \in W$. Similarly, if $X$ is normal (resp. reduced, irreducible, locally a complete intersection, Gorenstein, $\mathbb{Q}$-Gorenstein of index $r$, Cohen-Macaulay), then so is $X_{\mu}$ for general closed points $\mu \in \operatorname{Spec} B$. Also, $\operatorname{dim} X=\operatorname{dim} X_{\mu}$ and $\operatorname{codim}\left(Z_{i}, X\right)=\operatorname{codim}\left(Z_{i, \mu}, X_{\mu}\right)$ for general closed points $\mu \in \operatorname{Spec} B$. In particular, if $X$ is normal and $Z$ is a $\mathbb{Q}$-Weil (resp. $\mathbb{Q}$-Cartier) divisor on $X$, then $Z_{\mu}$ is a $\mathbb{Q}$-Weil (resp. $\mathbb{Q}$-Cartier) divisor on $X_{\mu}$ for general closed points $\mu \in \operatorname{Spec} B$. If $K_{X}$ is a canonical divisor on $X$, then $K_{X, \mu}$ gives a canonical divisor $K_{X_{\mu}}$ on $X_{\mu}$ for general closed points $\mu \in \operatorname{Spec} B$.

Given a morphism $f: X \rightarrow Y$ of schemes of finite type over $K$ and a model $\left(X_{B}, Y_{B}\right)$ of $(X, Y)$ over $B$, after possibly enlarging $B$, we may assume that $f$ is induced by a morphism $f_{B}: X_{B} \rightarrow Y_{B}$ of schemes of finite type over $B$. Given a closed point $\mu \in \operatorname{Spec} B$, we obtain a corresponding morphism $f_{\mu}: X_{\mu} \rightarrow Y_{\mu}$ of schemes of finite type over $\kappa(\mu)$. If $f$ is projective (resp. finite), then so is $f_{\mu}$ for general closed points $\mu \in \operatorname{Spec} B$.

Definition 2.1. Let $\boldsymbol{P}$ be a property defined for a triple $(X, D, Z)$, where $X$ is a scheme of finite type over a finite field, $D$ is an effective $\mathbb{Q}$-divisor on $X$ and $Z$ is an $\mathbb{R}_{\geq 0}$-linear combination of closed subschemes of $X$.

(i) $((X, D) ; Z)$ is said to be of $\boldsymbol{P}$ type if, for a model of $(X, D, Z)$ over a finitely generated $\mathbb{Z}$-subalgebra $B$ of $K$, there exists a dense open subset $W \subseteq \operatorname{Spec} B$ such that $\left(\left(X_{\mu}, D_{\mu}\right) ; Z_{\mu}\right)$ satisfies $\boldsymbol{P}$ for all closed points $\mu \in W$. 
(ii) $((X, D) ; Z)$ is said to be of dense $\boldsymbol{P}$ type if, for a model of $(X, D, Z)$ over a finitely generated $\mathbb{Z}$-subalgebra $B$ of $K$, there exists a dense subset of closed points $W \subseteq \operatorname{Spec} B$ such that $\left(\left(X_{\mu}, D_{\mu}\right) ; Z_{\mu}\right)$ satisfies $\boldsymbol{P}$ for all $\mu \in W$.

Remark 2.2. (1) By enlarging $B,((X, D) ; Z)$ is of $\boldsymbol{P}$ type if and only if for some model over $B, \boldsymbol{P}$ holds for all closed points $\mu \in \operatorname{Spec} B$.

(2) When $\boldsymbol{P}$ is strong $F$-regularity, pure $F$-regularity or sharp $F$-purity, the above definition is independent of the choice of a model.

There exists a correspondence between adjoint ideal sheaves and test ideal sheaves.

Theorem 2.3 ([Takagi 2008, Theorem 5.3]; cf. [Hara and Yoshida 2003; Takagi 2004b]). Let $X$ be a normal variety over a field $K$ of characteristic 0 , and let $Z=\sum_{i} t_{i} Z_{i}$ be a formal combination, where the $t_{i}$ are nonnegative real numbers and the $Z_{i}$ are proper closed subschemes of $X$. Let $D=\sum_{j} d_{j} D_{j}$ be a boundary divisor on $X$ such that $K_{X}+D$ is $\mathbb{Q}$-Cartier and no component of $\lfloor D\rfloor$ is contained in the support of the $Z_{i}$. Given any model of $(X, Z, D)$ over a finitely generated $\mathbb{Z}$-subalgebra $B$ of $K$, there exists a dense open subset $W \subseteq \operatorname{Spec} B$ such that

$$
\operatorname{adj}_{D}(X, Z)_{\mu}=\tilde{\tau}_{D_{\mu}}\left(X_{\mu}, Z_{\mu}\right)
$$

for every closed point $\mu \in W$. In particular, $((X, D) ; Z)$ is klt at $x$ if and only if it is of strongly $F$-regular type at $x$.

An analogous correspondence between $\log$ canonicity and $F$-purity, that is, the equivalence of $\log$ canonical pairs and pairs of dense sharply $F$-pure type, is largely conjectural.

Conjecture 2.4. Let $X$ be a normal variety over an algebraically closed field $K$ of characteristic 0 and $D$ be an effective $\mathbb{Q}$-divisor on $X$ such that $K_{X}+D$ is $\mathbb{Q}$-Cartier. Let $Z=\sum_{i} t_{i} Z_{i}$ be a formal combination, where the $t_{i}$ are nonnegative rational numbers and the $Z_{i}$ are proper closed subschemes of $X$. Fix an arbitrary point $x \in X$.

(i) $((X, D) ; Z)$ is log canonical at $x$ if and only if it is of dense sharply $F$-pure type at $x$.

(ii) Suppose that $(X, D)$ is log canonical at $x$. Given any model of $(X, D, Z, x)$ over a finitely generated $\mathbb{Z}$-subalgebra $B$ of $K$, there exists a dense subset of closed points $W \subseteq$ Spec $B$ such that

$$
\operatorname{lct}_{x}((X, D) ; Z)=\mathrm{fpt}_{x_{\mu}}\left(\left(X_{\mu}, D_{\mu}\right) ; Z_{\mu}\right)
$$

for all $\mu \in W$. 
Remark 2.5. (1) It is easy to see that (i) implies (ii) in Conjecture 2.4.

(2) If $((X, D) ; Z)$ is of dense sharply $F$-pure type at $x$, then by [Hara and Watanabe 2002, Theorem 3.3; Takagi 2004a, Proposition 3.8], it is log canonical at $x$.

Remark 2.6. Conjecture 2.4 is known to hold in the following cases (see also Theorem 4.1):

(i) $X$ is a $\mathbb{Q}$-Gorenstein toric variety, $D=0$ and the $Z_{i}$ are monomial subschemes.

(ii) $X$ is the affine space $\mathbb{A}_{K}^{n}, D=0$ and $Z=t_{1} Z_{1}$, where $Z_{1}$ is a binomial complete intersection subscheme or a space monomial curve (in the latter case, $n=3$ ).

(iii) $X$ is a normal surface, $D$ is an integral effective divisor on $X$ and $Z=\varnothing$.

(iv) $X$ is the affine space $\mathbb{A}_{K}^{n}, D=0$ and $Z$ is a hypersurface of $X$ such that the coefficients of terms of its defining equation are algebraically independent over $\mathbb{Q}$.

Case (i) follows from [Blickle 2004, Theorem 3], (ii) from [Shibuta and Takagi 2009, Theorem 0.1] and (iv) from [Hernández 2011, Theorem 5.16]. We explain here how to check the case (iii). If $D \neq 0$, then it follows from comparing [Hara and Watanabe 2002, Theorem 4.5] with [Kawamata 1988, Theorem 9.6]. So we consider the case when $D=0$. By Remark 2.5, it suffices to show that a twodimensional $\log$ canonical singularity $(X, x)$ is of dense $F$-pure type. Passing to an index-1 cover, we may assume that $(X, x)$ is Gorenstein. If it is log terminal, then by [Hara 1998, Theorem 5.2] (see also Theorem 2.3), it is of $F$-regular type and, in particular, of dense $F$-pure type. Hence, we can assume that $(X, x)$ is not $\log$ terminal, that is, $(X, x)$ is a cusp singularity or a simple elliptic singularity. By [Mehta and Srinivas 1991, Theorem 1.2; Watanabe 1988, Theorem 1.7], cusp singularities are of dense $F$-pure type. Also, by [Mehta and Srinivas 1991], a simple elliptic singularity with exceptional elliptic curve $E$ is of dense $F$-pure type if and only if for a model $E_{B}$ of $E$ over a finitely generated $\mathbb{Z}$-subalgebra $B \subseteq K$, there exists a dense subset of closed points $W \subseteq \operatorname{Spec} B$ such that $E_{\mu}$ is ordinary for all $\mu \in W$. Applying the same argument as the proof of [Mustaţă and Srinivas 2011, Proposition 5.3], we may assume that $E$ is defined over $\overline{\mathbb{Q}}$. It then follows from the ordinary reduction theorem of Serre [1966] that such $W$ always exists. Thus, simple elliptic singularities are of dense $F$-pure type.

Lemma 2.7. In order to prove Conjecture 2.4 , it is enough to consider the case when $Z=\varnothing$.

Proof. Since the question is local, we work in a sufficiently small neighborhood of $x$. By Remark 2.5, it suffices to show that if $((X, D) ; Z)$ is $\log$ canonical, then it is of dense sharply $F$-pure type. 
Suppose that $((X, D) ; Z)$ is $\log$ canonical. Let $h_{i, 1}, \ldots, h_{i, m_{i}}$ be a system of generators for $\mathscr{I}_{Z_{i}}$ for each $i$. Let $g_{i, 1}, \ldots, g_{i, m_{i}}$ be general linear combinations of $h_{i, 1}, \ldots, h_{i, m_{i}}$ with coefficients in $K$, and set $g_{i}:=\prod_{j=1}^{m_{i}} g_{i, j}$ so that

$$
\left(X, D+\sum_{i} \frac{t_{i}}{m_{i}} \operatorname{div}_{X}\left(g_{i}\right)\right)
$$

is $\log$ canonical. On the other hand, since $g_{i} \in \Im_{Z_{i}}^{m_{i}}$, if $(\dagger)$ is of dense sharply $F$-pure type, then so is $((X, D) ; Z)$. Therefore, it is enough to show that the log canonical pair $(\dagger)$ is of dense sharply $F$-pure type.

Mustață and Srinivas [2011] recently proposed the following more arithmetic conjecture and related it to another conjecture on a comparison between multiplier ideal sheaves and test ideal sheaves:

Conjecture 2.8 [Mustaţă and Srinivas 2011, Conjecture 1.1]. Let $X$ be an ndimensional smooth projective variety over $\overline{\mathbb{Q}}$. Given a model of $X$ over a finitely generated $\mathbb{Z}$-subalgebra $B$ of $\overline{\mathbb{Q}}$, there exists a dense subset of closed points $W \subseteq$ Spec $B$ such that the action induced by Frobenius on $H^{n}\left(X_{\mu}, \mathcal{O}_{X_{\mu}}\right)$ is bijective for all $\mu \in W$.

Remark 2.9. Conjecture 2.8 is known to be true when $X$ is a smooth projective curve of genus less than or equal to 2 (see [Mustaţă and Srinivas 2011, Example 5.5], which can be traced back to [Ogus 1982; Serre 1966]) or a smooth projective surface of Kodaira dimension 0; see [Jang 2011, Proposition 2.3].

Example 2.10. We check that Conjecture 2.8 holds for the Fermat hypersurface $X$ of degree $d$ in $\mathbb{P}_{K}^{n}$ over a field $K$ of characteristic 0 . Given a prime number $p$, set $S_{p}:=\mathbb{F}_{p}\left[x_{0}, \ldots, x_{n}\right], \mathfrak{m}_{p}:=\left(x_{0}, \ldots, x_{n}\right) \subseteq S_{p}, f_{p}:=x_{0}^{d}+\cdots+x_{n}^{d} \in S_{p}$ and $X_{p}:=\operatorname{Proj} S_{p} / f_{p}$. Since $H^{n-1}\left(X_{p}, \mathcal{O}_{X_{p}}\right)=0$ for almost all $p$ when $d \leq n$, we consider the case when $d \geq n+1$. Note that

$$
H^{n-1}\left(X_{p}, O_{X_{p}}\right) \cong\left[H_{\mathfrak{m}_{p}}^{n}\left(S_{p} / f_{p}\right)\right]_{0} \cong\left[\left(0: f_{p}\right)_{H_{\mathfrak{m}_{p}}^{n+1}\left(S_{p}\right)}\right]_{-d} .
$$

Via this isomorphism, the action induced by Frobenius on $H^{n-1}\left(X_{p}, \mathcal{O}_{X_{p}}\right)$ is identified with

$$
f_{p}^{p-1} F:\left[\left(0: f_{p}\right)_{H_{\mathfrak{m} p}^{n+1}\left(S_{p}\right)}\right]_{-d} \rightarrow\left[\left(0: f_{p}\right)_{H_{\mathfrak{m} p}^{n+1}\left(S_{p}\right)}\right]_{-d},
$$

where $F: H_{\mathfrak{m}_{p}}^{n+1}\left(S_{p}\right) \rightarrow H_{\mathfrak{m}_{p}}^{n+1}\left(S_{p}\right)$ is the map induced by Frobenius on $H_{\mathfrak{m}_{p}}^{n+1}\left(S_{p}\right)$. Let

$$
\xi:=\left[z /\left(x_{0} \cdots x_{n}\right)^{m}\right] \in H_{\mathfrak{m}_{p}}^{n+1}\left(S_{p}\right)
$$

be a homogeneous element such that $f_{p}^{p-1} F(\xi)$ vanishes; that is, such that $f_{p}^{p-1} z^{p}$ lies in $\left(x_{0}^{m p}, \ldots, x_{n}^{m p}\right)$. Set $W:=\{p \in \operatorname{Spec} \mathbb{Z} \mid p \equiv 1 \bmod d\}$, which is a dense 
subset of Spec $\mathbb{Z}$, and suppose that $p \in W$. Let $a_{0}, \ldots, a_{n}$ be nonnegative integers such that $\sum_{i=0}^{n} a_{n}=d-n-1$. Then the term

$$
\left(x_{0}^{d\left(a_{0}+1\right)} \cdots x_{n}^{d\left(a_{n}+1\right)}\right)^{(p-1) / d}=\left(x_{0}^{a_{0}} \cdots x_{n}^{a_{n}}\right)^{p} x_{0}^{p-a_{0}-1} \cdots x_{n}^{p-a_{n}-1}
$$

appears in the expansion of $f_{p}^{p-1}$. Since $\left\{x_{1}^{i_{1}} \cdots x_{n}^{i_{n}}\right\}_{0 \leq i_{1}, \ldots, i_{n} \leq p-1}$ is a free basis of $S_{p}$ as an $S_{p}^{p}$-module, $f^{p-1} z^{p}$ can be written as

$$
f^{p-1} z^{p}=u\left(x_{0}^{a_{0}} \cdots x_{n}^{a_{n}} z\right)^{p} x_{0}^{p-a_{0}-1} \cdots x_{n}^{p-a_{n}-1}+\sum_{i_{j} \neq p-a_{j}-1} g_{i_{0}, \ldots, i_{n}}^{p} x_{0}^{i_{0}} \cdots x_{n}^{i_{n}},
$$

where $u \in \mathbb{F}_{p}$ is a nonzero element and $g_{i_{0}, \ldots, i_{n}} \in S_{p}$ for each $0 \leq i_{0}, \ldots, i_{n} \leq p-1$. Let $\varphi: F_{*} S_{p} \rightarrow S_{p}$ be the $S$-linear map sending $x_{0}^{p-a_{0}-1} \cdots x_{n}^{p-a_{n}-1}$ to 1 and the other part of the basis to 0 . Then

$$
u x_{0}^{a_{0}} \cdots x_{n}^{a_{n}} z=\varphi\left(f^{p-1} z^{p}\right) \in \varphi\left(\left(x_{0}^{m p}, \ldots, x_{n}^{m p}\right)\right) \subseteq\left(x_{0}^{m}, \ldots, x_{n}^{m}\right) .
$$

By the definition of the $a_{i}$, one has $\mathfrak{m}_{p}^{d-n-1} z \subseteq\left(x_{0}^{m}, \ldots, x_{n}^{m}\right)$, that is, $\mathfrak{m}_{p}^{d-n-1} \xi=0$ in $H_{\mathfrak{m}_{p}}^{n+1}\left(S_{p}\right)$. This means that $\operatorname{deg} \xi \geq-d+1$, and we conclude that, for all $p \in W$, $f_{p}^{p-1^{\mathfrak{m}}} F:\left[\left(0: f_{p}\right)_{H_{\mathfrak{m} p}^{n+1}\left(S_{p}\right)}\right]_{-d} \rightarrow\left[\left(0: f_{p}\right)_{H_{\mathfrak{m}_{p}}^{n+1}\left(S_{p}\right)}\right]_{-d}$ is injective.

The following result comes from a discussion with Karl Schwede, whom the author thanks:

Theorem 2.11. If Conjecture 2.8 holds, then Conjecture 2.4 holds as well.

To prove it, we use a notion of sharp $F$-purity for noneffective integral divisors.

Definition 2.12. Let $X$ be an $F$-finite normal integral scheme of characteristic $p>0$ and $D$ be a (not necessarily effective) integral divisor on $X$. We assume that $K_{X}+D$ is $\mathbb{Q}$-Cartier with index not divisible by $p$. Let $x \in X$ be an arbitrary point. We decompose $D$ as $D=D_{+}-D_{-}$, where $D_{+}$and $D_{-}$are effective integral divisors on $X$ that have no common irreducible components. We then say that the pair $(X, D)$ is sharply $F$-pure at $x$ if there exists an integer $e_{0}>0$ such that $\left(p^{e_{0}}-1\right)\left(K_{X}+D\right)$ is Cartier and that for all positive multiples $e=n e_{0}$ of $e_{0}$, one has an $\mathcal{O}_{X, x}$-linear map $\varphi: F_{*}^{e} \mathscr{O}_{X}\left(\left(p^{e}-1\right) D_{+}+D_{-}\right)_{x} \rightarrow \mathcal{O}_{X}\left(D_{-}\right)_{x}$ whose image of $F_{*}^{e} O_{X}\left(D_{-}\right)_{x}$ contains 1 . We say that $(X, D)$ is sharply $F$-pure if it is sharply $F$-pure at every closed point of $X$.

If $D$ is an effective integral divisor, this definition coincides with Definition 1.3(ii). We need a variant of [Schwede and Tucker 2012, Theorem 6.28] involving sharp $F$-purity in the sense of Definition 2.12.

Lemma 2.13 (cf. [Schwede and Tucker 2012, Theorem 6.28]). Let $\pi: Y \rightarrow X$ be a finite separable morphism of $F$-finite normal integral schemes of characteristic $p>0$. Let $\Delta_{X}$ be an effective $\mathbb{Q}$-divisor on $X$ such that $K_{X}+\Delta_{X}$ is $\mathbb{Q}$-Cartier with index not divisible by $p$. Suppose that $\Delta_{Y}$ is an integral divisor on $Y$ such that 
$K_{Y}+\Delta_{Y}=\pi^{*}\left(K_{X}+\Delta_{X}\right)$. Also, we assume that the trace map $\operatorname{Tr}_{Y / X}: \pi_{*} \mathrm{O}_{Y} \rightarrow \mathrm{O}_{X}$ is surjective. Then $\left(X, \Delta_{X}\right)$ is sharply $F$-pure if and only if $\left(Y, \Delta_{Y}\right)$ is sharply $F$-pure in the sense of Definition 2.12.

Proof. The statement is local on $X$, so we assume that $X=\operatorname{Spec} A$ and $Y=\operatorname{Spec} B$, where $A$ is a local ring and $B$ is a semilocal ring. There exists $e_{0} \in \mathbb{N}$ such that $\left(p^{e_{0}}-1\right)\left(K_{X}+\Delta\right)$ is Cartier. Then $\operatorname{Hom}_{A}\left(F_{*}^{e} A\left(\left(p^{e}-1\right) \Delta_{X}\right), A\right)$ is a free $F_{*}^{e} A$ module of rank 1 for all positive multiples $e=n e_{0}$ of $e_{0}$. Let $\varphi_{X}: F_{*}^{e} A \rightarrow A$ be its generator. We decompose $\Delta_{Y}$ as $\Delta_{Y,+}-\Delta_{Y,-}$, where $\Delta_{Y,+}$ and $\Delta_{Y,-}$ are effective integral divisors on $Y$ that have no common components. Then the $F_{*}^{e} B$-module

$$
\begin{aligned}
\operatorname{Hom}_{B}\left(F_{*}^{e} B\left(\left(p^{e}-1\right) \Delta_{Y,+}+\Delta_{Y,-}\right), B\left(\Delta_{Y,-}\right)\right) & \cong F_{*}^{e} B\left(\left(1-p^{e}\right)\left(K_{Y}+\Delta_{Y}\right)\right) \\
& =F_{*}^{e} \pi^{*} A\left(\left(1-p^{e}\right)\left(K_{X}+\Delta_{X}\right)\right) \\
& \cong F_{*}^{e} \pi^{*} A=F_{*}^{e} B,
\end{aligned}
$$

and we pick its generator $\varphi_{Y}: F_{*}^{e} B\left(\Delta_{Y,-}\right) \rightarrow B\left(\Delta_{Y,-}\right)$ extending $\varphi_{X}: F_{*}^{e} A \rightarrow A$.

Suppose that $\left(X, \Delta_{X}\right)$ is sharply $F$-pure. By the definition of sharp $F$-purity, after possibly enlarging $e$, we have that $1 \in \operatorname{Im} \varphi_{X} \subseteq \operatorname{Im} \varphi_{Y}$, and hence, $\left(Y, \Delta_{Y}\right)$ is sharply $F$-pure.

Conversely, suppose that $\left(Y, \Delta_{Y}\right)$ is sharply $F$-pure. Making $e$ larger if necessary, we may assume that $1 \in \operatorname{Im} \varphi_{Y}$. Note that $\Delta_{Y}=\pi^{*} \Delta_{X}-R$ and $R \geq \Delta_{Y,-}$, where $R$ denotes the ramification divisor of $\pi$. Then the $F_{*}^{e} B$-module

$$
\begin{aligned}
\operatorname{Hom}_{B}\left(F_{*}^{e} B\left(\left(p^{e}-1\right) \pi^{*} \Delta_{X}+R\right)\right. & , B(R)) \\
& \cong F_{*}^{e} \pi^{*} A\left(\left(1-p^{e}\right)\left(K_{X}+\Delta_{X}\right)\right) \\
& \cong \operatorname{Hom}_{B}\left(F_{*}^{e} B\left(\left(p^{e}-1\right) \Delta_{Y,+}+\Delta_{Y,-}\right), B\left(\Delta_{Y,-}\right)\right) .
\end{aligned}
$$

We pick its generator $\widetilde{\varphi}_{Y}: F_{*}^{e} B(R) \rightarrow B(R)$ extending $\varphi_{Y}: F_{*}^{e} B\left(\Delta_{Y,-}\right) \rightarrow B\left(\Delta_{Y,-}\right)$. Since the trace map $\operatorname{Tr}_{Y / X}$ corresponds to the ramification divisor $R$, we have the following commutative diagram:

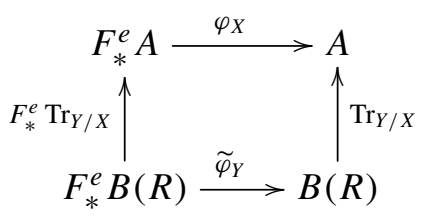

The surjectivity of the trace map $\operatorname{Tr}_{Y / X}: B \rightarrow A$ implies that

$$
1 \in \operatorname{Tr}_{Y / X}\left(\operatorname{Im} \varphi_{Y}\right) \subseteq \operatorname{Tr}_{Y / X}\left(\operatorname{Im} \tilde{\varphi}_{Y}\right)=\varphi_{X}\left(\operatorname{Im} F_{*}^{e} \operatorname{Tr}_{Y / X}\right)=\operatorname{Im} \varphi_{X}
$$

because $B \subseteq \operatorname{Im} \varphi_{Y}$. Thus, $\left(X, \Delta_{X}\right)$ is sharply $F$-pure.

Proof of Theorem 2.11. Let the notation be as in Conjecture 2.4. By Lemma 2.7, we may assume that $K_{X}+D$ is Cartier and $Z=\varnothing$. Since the question is local, we 
work in a sufficiently small neighborhood of $x$. By Remark 2.5, it suffices to show that if $(X, D)$ is log canonical, then it is of dense sharply $F$-pure type.

Suppose that $(X, D)$ is log canonical. By [Kollár and Mori 1998, Section 2.4], there exists a finite morphism $f: X^{\prime} \rightarrow X$ from a normal variety $X^{\prime}$ over $K$ such that $f^{*}\left(K_{X}+D\right)$ is Cartier. Let $D^{\prime}$ be a (not necessarily effective) integral divisor on $X^{\prime}$ such that $K_{X^{\prime}}+D^{\prime}=f^{*}\left(K_{X}+D\right)$. It then follows from [Kollár and Mori 1998, Proposition 5.20] that $\left(X^{\prime}, D^{\prime}\right)$ is log canonical. We decompose $D^{\prime}$ as $D^{\prime}=D_{+}^{\prime}-D_{-}^{\prime}$, where $D_{+}^{\prime}$ and $D_{-}^{\prime}$ are effective integral divisors on $X$ that have no common components. Take a $\log$ resolution $\pi: \widetilde{X} \rightarrow X^{\prime}$ of $\left(X^{\prime}, D^{\prime}\right)$, and let $E$ denote the reduced divisor supported on the $\pi$-exceptional locus $\operatorname{Exc}(\pi)$. Let $\left(X_{B}, D_{B}, X_{B}^{\prime}, D_{B}^{\prime}=D_{+, B}^{\prime}-D_{-, B}^{\prime}, \pi_{B}, E_{B}\right)$ be a model of $\left(X, D, X^{\prime}, D^{\prime}=\right.$ $\left.D_{+}^{\prime}-D_{-}^{\prime}, \pi, E\right)$ over a finitely generated $\mathbb{Z}$-subalgebra $B$ of $K$. After possibly enlarging $B$, we may assume that $K_{X_{\mu}}+D_{\mu}$ is $\mathbb{Q}$-Cartier with index not divisible by the characteristic $p(\mu)$ and that the trace map $\operatorname{Tr}_{X_{\mu}^{\prime} / X_{\mu}}: f_{\mu_{*}} \mathcal{O}_{X_{\mu}^{\prime}} \rightarrow \mathcal{O}_{X_{\mu}}$ is surjective for all closed points $\mu \in \operatorname{Spec} B$.

By virtue of [Mustaţă and Srinivas 2011, Theorem 5.10], there exists a dense subset of closed points $W \subseteq \operatorname{Spec} B$ such that for every integer $e \geq 1$ and every $\mu \in W$, the map

$$
\pi_{\mu_{*}} F_{*}^{e}\left(\mathbb{O}_{\widetilde{X}_{\mu}}\left(K_{\widetilde{X}_{\mu}}+\pi_{\mu_{*}}^{-1} D_{+, \mu}^{\prime}+E_{\mu}\right)\right) \rightarrow \pi_{\mu_{*}} \mathrm{O}_{\widetilde{X}_{\mu}}\left(K_{\widetilde{X}_{\mu}}+\pi_{\mu_{*}}^{-1} D_{+, \mu}^{\prime}+E_{\mu}\right),
$$

induced by the canonical dual of the $e$-times iterated Frobenius map $\mathbb{O}_{\tilde{X}_{\mu}} \rightarrow F_{*}^{e} \mathcal{O}_{\tilde{X}_{\mu}}$, is surjective. Tensoring $(\diamond)$ with $0_{X_{\mu}^{\prime}}\left(-K_{X_{\mu}^{\prime}}-D_{\mu}^{\prime}\right)$, one can see that the map

$$
\rho: \pi_{\mu_{*}} F_{*}^{e}\left(\mathrm{O}_{\widetilde{X}_{\mu}}\left(M+\left(1-p(\mu)^{e}\right) \pi_{\mu}^{*}\left(K_{X_{\mu}^{\prime}}+D_{\mu}^{\prime}\right)\right)\right) \rightarrow \pi_{\mu_{*}} \mathrm{O}_{\widetilde{X}_{\mu}}(M)
$$

is surjective, where $M=K_{\widetilde{X}_{\mu}}+\pi_{\mu_{*}}^{-1} D_{+, \mu}^{\prime}-\pi_{\mu}^{*}\left(K_{X_{\mu}^{\prime}}+D_{\mu}^{\prime}\right)+E_{\mu}$. Since $\left(X^{\prime}, D^{\prime}\right)$ is log canonical, $1 \in \pi_{\mu_{*}} \mathrm{O}_{X_{\mu}}(M) \subseteq \mathcal{O}_{X_{\mu}^{\prime}}\left(D_{-, \mu}^{\prime}\right)$. By Grothendieck duality, $\rho$ is identified with the evaluation map

$$
\begin{aligned}
& F_{*}^{e} \mathscr{O}_{X_{\mu}^{\prime}}\left(D_{-, \mu}^{\prime}\right) \otimes \mathcal{H o m}_{\mathrm{O}_{X_{\mu}^{\prime}}}\left(F_{*}^{e} \mathcal{O}_{X_{\mu}^{\prime}}\left(\left(p(\mu)^{e}-1\right) D_{+, \mu}^{\prime}+D_{-, \mu}^{\prime}\right), \mathcal{O}_{X_{\mu}^{\prime}}\left(D_{-, \mu}^{\prime}\right)\right) \\
& \rightarrow \mathcal{O}_{X_{\mu}^{\prime}}\left(D_{-, \mu}^{\prime}\right) .
\end{aligned}
$$

The subjectivity of $\rho$ then implies that there exists an $0_{X^{\prime}}$-linear map

$$
\varphi_{X^{\prime}}: F_{*}^{e} \mathcal{O}_{X_{\mu}^{\prime}}\left(\left(p(\mu)^{e}-1\right) D_{+, \mu}^{\prime}+D_{-, \mu}^{\prime}\right) \rightarrow \mathcal{O}_{X_{\mu}^{\prime}}\left(D_{-, \mu}^{\prime}\right)
$$

such that $1 \in \varphi_{X^{\prime}}\left(F_{*}^{e} \mathcal{O}_{X_{\mu}^{\prime}}\left(D_{-, \mu}^{\prime}\right)\right)$. That is, $\left(X_{\mu}^{\prime}, D_{\mu}^{\prime}\right)$ is sharply $F$-pure in the sense of Definition 2.12. Applying Lemma 2.13, we conclude that $\left(X_{\mu}, D_{\mu}\right)$ is sharply $F$-pure for all $\mu \in W$.

Remark 2.14. Let $Y$ be an $S 2, G 1$ and seminormal variety over an algebraically closed field $K$ of characteristic 0 and $\Gamma$ be an effective $\mathbb{Q}$-Weil divisorial sheaf on $Y$ such that $K_{Y}+\Gamma$ is $\mathbb{Q}$-Cartier. Combining Theorem 2.11 with [Miller and 
Schwede 2012, Corollary 4.4], we can conclude that if Conjecture 2.8 holds, then the pair $(Y, \Gamma)$ is semilog canonical if and only if it is of dense sharply $F$-pure type.

\section{Restriction theorem for adjoint ideal sheaves}

In this section, building on an earlier work [Takagi 2010], we give a new proof of Eisenstein's restriction theorem for adjoint ideal sheaves using test ideal sheaves.

Definition 3.1. Let $A$ be a smooth variety over an algebraically closed field $K$ of characteristic 0 and $X \subseteq A$ be a normal $\mathbb{Q}$-Gorenstein closed subvariety of codimension $c$. Let $r$ denote the Gorenstein index of $X$, that is, the smallest positive integer $m$ such that $m K_{X}$ is Cartier. Then the l.c.i. defect ideal sheaf ${ }^{1} J_{X} \subseteq \mathcal{O}_{X}$ is defined as follows. Since the construction is local, we may consider the germ at a closed point $x \in X \subseteq A$. We take generally a closed subscheme $Y$ of $A$ that contains $X$ and is locally a complete intersection (l.c.i. for short) of codimension $c$. By Bertini's theorem, $Y$ is the scheme-theoretic union of $X$ and another variety $C^{Y}$ of codimension $c$. Then the closed subscheme $D^{Y}:=\left.C^{Y}\right|_{X}$ of $X$ is a Weil divisor such that $r D^{Y}$ is Cartier and $\mathscr{O}_{X}\left(r K_{X}\right)=\mathscr{O}_{X}\left(-r D^{Y}\right) \omega_{Y}^{\otimes r}$. The 1.c.i. defect ideal sheaf $J_{X}$ is defined by

$$
J_{X}:=\sum_{Y} \mathfrak{O}_{X}\left(-r D^{Y}\right)
$$

where $Y$ runs through all the general l.c.i. closed subschemes of codimension $c$ containing $X$. Note that the support of $J_{X}$ exactly coincides with the non-l.c.i. locus of $X$. In particular, $J_{X}=\mathscr{O}_{X}$ if and only if $X$ is l.c.i. The reader is referred to [Kawakita 2008, Section 2; Ein and Mustaţă 2009, Section 9.2] for further properties of l.c.i. defect ideal sheaves.

Now we give a new proof of the theorem of Eisenstein [2010, Corollary 5.2].

Theorem 3.2. Let $A$ be a smooth variety over an algebraically closed field $K$ of characteristic 0 and $Z=\sum_{i=1}^{m} t_{i} Z_{i}$ be a formal combination, where the $t_{i}$ are nonnegative real numbers and the $Z_{i}$ are proper closed subschemes of $A$. If $X$ is a normal $\mathbb{Q}$-Gorenstein closed subvariety of $A$ that is not contained in the support of any $Z_{i}$, then

$$
\mathscr{g}\left(X,\left.Z\right|_{X}+\frac{1}{r} V\left(J_{X}\right)\right)=\left.\operatorname{adj}_{X}(A, Z)\right|_{X},
$$

where $r$ is the Gorenstein index of $X$ and $J_{X}$ is the l.c.i. defect ideal sheaf of $X$.

Proof. We refine the proof of [Takagi 2010, Theorem 3.1]. The inclusion

\footnotetext{
${ }^{1}$ We follow a construction due to Kawakita [2008], but our terminology is slightly different from his. We warn the reader that the ideal sheaf called the 1.c.i. defect ideal in [Kawakita 2008] is different from our $J_{X}$. Also, Ein and Mustață [2009] introduced a very similar ideal, which coincides with our $J_{X}$ up to integral closure.
} 


$$
\left.\mathscr{F}\left(X,\left.Z\right|_{X}+\frac{1}{r} V\left(J_{X}\right)\right) \supseteq \operatorname{adj}_{X}(A, Z)\right|_{X}
$$

follows from a combination of [Ein and Mustaţă 2009, Remark 8.5] and [Takagi 2010, Lemma 1.7]. Hence, we will prove the converse inclusion.

We consider the germ at a closed point $x \in X \cap \bigcap_{i=1}^{m} Z_{i} \subset A$ since the question is local. Let $c$ denote the codimension of $X$ in $A$. Take generally a subscheme $Y$ of $A$ that contains $X$ and is 1.c.i. of codimension $c$, so $Y$ is the scheme-theoretic union of $X$ and a variety $C^{Y}$. Then $D^{Y}:=\left.C^{Y}\right|_{X}$ is a Weil divisor on $X$ such that $r D^{Y}$ is Cartier. By a general choice of $Y$, one has

$$
\mathscr{F}\left(X,\left.Z\right|_{X}+\frac{1}{r} V\left(J_{X}\right)\right)=\operatorname{adj}_{D^{Y}}\left(X,\left.Z\right|_{X}\right)
$$

(which follows from an argument similar to the claim in the proof of [Takagi 2010, Theorem 3.1]). Therefore, it is enough to show that $\left.\operatorname{adj}_{D^{Y}}\left(X,\left.Z\right|_{X}\right) \subseteq \operatorname{adj}_{X}(A, Z)\right|_{X}$.

By Theorem 2.3 and [Takagi 2010, Theorem 2.7], in order to prove this inclusion, it suffices to show that given any model of $\left(A, X, Y, Z, C^{Y}, D^{Y}\right)$ over a finitely generated $\mathbb{Z}$-subalgebra $B$ of $K$, one has

$$
\left.\tilde{\tau}_{D_{\mu}^{Y}}\left(X_{\mu},\left.Z_{\mu}\right|_{X_{\mu}}\right) \subseteq \tilde{\tau}_{X_{\mu}}\left(A_{\mu}, Z_{\mu}\right)\right|_{X_{\mu}}
$$

for general closed points $\mu \in \operatorname{Spec} B$. Since $\mu$ is a general point of $\operatorname{Spec} B$ and the formation of test ideal sheaves commutes with localization, we may assume that $\mathrm{O}_{A_{\mu}}$ is an $F$-finite regular local ring of characteristic $p=p(\mu)>r, X_{\mu}=V(I)$ is a normal $\mathbb{Q}$-Gorenstein closed subscheme of $A_{\mu}$ with Gorenstein index $r$ and $Y_{\mu}=V\left(\left(f_{1}, \ldots, f_{c}\right)\right)$ is a complete intersection closed subscheme of codimension $c$ containing $X_{\mu}$. We may assume in addition that $D_{\mu}^{Y}$ is a Weil divisor on $X_{\mu}$ such that $r D_{\mu}^{Y}$ is Cartier and $\mathcal{O}_{X_{\mu}}\left(r K_{X_{\mu}}\right)=\mathcal{O}_{X_{\mu}}\left(-r D_{\mu}^{Y}\right) \omega_{Y_{\mu}}^{\otimes r}$. We take a germ $g \in \mathcal{O}_{A_{\mu}}$ whose image $\bar{g}$ is the local equation of $r D_{\mu}^{Y}$ on $\mathcal{O}_{X_{\mu}}$. Let $\mathfrak{a}_{i} \subseteq \mathcal{O}_{A_{\mu}}$ be the defining ideal of $Z_{i, \mu}$ for each $i=1, \ldots, m$. Fix an integer $e_{0} \geq 1$ such that $p^{e_{0}}-1$ is divisible by $r$, and set $q_{0}:=p^{e_{0}}$.

Claim. For all powers $q=q_{0}^{n}$ of $q_{0}$, one has

$$
g^{(q-1) / r}\left(I^{[q]}: I\right)=\left(f_{1} \cdots f_{c}\right)^{q-1} \text { in } 0_{A_{\mu}} / I^{[q]} .
$$

Proof. Since $q-1$ is divisible by $r$,

$$
\begin{aligned}
\mathrm{O}_{X_{\mu}}\left((1-q)\left(K_{X_{\mu}}+D_{\mu}^{Y}\right)\right) & =\left.\mathcal{O}_{Y_{\mu}}\left((1-q) K_{Y_{\mu}}\right)\right|_{X_{\mu}} \\
& =\left.\mathcal{O}_{A_{\mu}}\left((1-q)\left(K_{A_{\mu}}+\sum_{i=1}^{c} \operatorname{div}_{A_{\mu}}\left(f_{i}\right)\right)\right)\right|_{X_{\mu}} .
\end{aligned}
$$

Set $e:=n e_{0}$. By making use of Grothendieck duality, this implies that the natural map of $F_{*}^{e} \mathrm{O}_{A_{\mu}}$-modules

$\operatorname{Hom}_{\mathbb{O}_{A_{\mu}}}\left(F_{*}^{e} \mathcal{O}_{A_{\mu}}\left((q-1) \sum_{i=1}^{c} \operatorname{div}_{A_{\mu}}\left(f_{i}\right)\right), \mathcal{O}_{A_{\mu}}\right) \rightarrow \operatorname{Hom}_{\mathscr{O}_{X_{\mu}}}\left(F_{*}^{e} \mathcal{O}_{X_{\mu}}\left((q-1) D_{\mu}^{Y}\right), \widehat{O}_{X_{\mu}}\right)$ 
induced by restriction is surjective. It then follows from Lemma 1.5(1) that the $\mathrm{O}_{A_{\mu}}$-linear map

$$
\left(f_{1} \cdots f_{c}\right)^{q-1} \widehat{O}_{A_{\mu}} \rightarrow \frac{g^{(q-1) / r}\left(I^{[q]}: I\right)}{I^{[q]}}
$$

induced by the natural quotient map $0_{A_{\mu}} \rightarrow \mathcal{O}_{A_{\mu}} / I^{[q]}$ is surjective. Thus, we obtain the assertion.

Let $\varphi_{X_{\mu}, e_{0}}: F_{*}^{n e_{0}} \mathrm{O}_{X_{\mu}} \rightarrow \mathcal{O}_{X_{\mu}}$ be a generator for the rank-1 free $F_{*}^{n e_{0}} \mathcal{O}_{X_{\mu}}$-module $\operatorname{Hom}_{\mathfrak{O}_{X_{\mu}}}\left(F_{*}^{n e_{0}} \mathrm{O}_{X_{\mu}}, \mathcal{O}_{X_{\mu}}\right)$. Then $\tilde{\tau}_{D_{\mu}^{Y}}\left(X_{\mu},\left.Z_{\mu}\right|_{X_{\mu}}\right)$ is the unique smallest ideal $J$ whose support does not contain any component of $D_{\mu}^{Y}$ and that satisfies

$$
\varphi_{X_{\mu}, n e_{0}}\left(F_{*}^{n e_{0}}\left(J g^{\left(q_{0}^{n}-1\right) / r} \mathfrak{a}_{1}^{\left\lceil t_{1}\left(q_{0}^{n}-1\right)\right\rceil} \cdots \mathfrak{a}_{m}^{\left\lceil t_{m}\left(q_{0}^{n}-1\right)\right\rceil}\right)\right) \subseteq J
$$

for all integers $n \geq 1$. By Lemma 1.5(1), there exist an $\mathscr{O}_{A_{\mu}}$-linear map

$$
\varphi_{A_{\mu}, n e_{0}}: F_{*}^{n e_{0}} \mathrm{O}_{A_{\mu}} \rightarrow \mathrm{O}_{A_{\mu}}
$$

and a germ $h_{n} \in \mathrm{O}_{A_{\mu}}$ whose image is a generator for the cyclic $\mathrm{O}_{X_{\mu}}$-module $\left(I^{\left[q_{0}^{n}\right]}: I\right) / I^{\left[q_{0}\right]}$ such that we have the commutative diagram

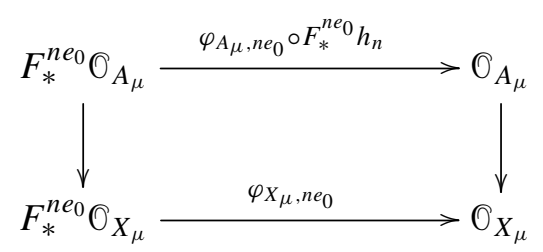

where the vertical maps are natural quotient maps. By the definition of $\tilde{\tau}_{X_{\mu}}\left(A_{\mu}, Z_{\mu}\right)$, one has

$$
\varphi_{A_{\mu}, n e_{0}}\left(F_{*}^{n e_{0}}\left(\tilde{\tau}_{X_{\mu}}\left(A_{\mu}, Z_{\mu}\right) I^{c\left(q_{0}^{n}-1\right)} \mathfrak{a}_{1}^{\left\lceil t_{1}\left(q_{0}^{n}-1\right)\right\rceil} \cdots \mathfrak{a}_{m}^{\left\lceil t_{m}\left(q_{0}^{n}-1\right)\right\rceil}\right)\right) \subseteq \tilde{\tau}_{X_{\mu}}\left(A_{\mu}, Z_{\mu}\right) .
$$

Since $g^{\left(q_{0}^{n}-1\right) / r} h_{n} \in I^{c\left(q_{0}^{n}-1\right)}+I^{\left[q_{0}^{n}\right]}$ by the claim,

$\varphi_{A_{\mu}, n e_{0}}\left(F_{*}^{n e_{0}}\left(\tilde{\tau}_{X_{\mu}}\left(A_{\mu}, Z_{\mu}\right) g^{\left(q_{0}^{n}-1\right) / r} h_{n} \mathfrak{a}_{1}^{\left\lceil t_{1}\left(q_{0}^{n}-1\right)\right\rceil} \cdots \mathfrak{a}_{m}^{\left\lceil t_{m}\left(q_{0}^{n}-1\right)\right\rceil}\right)\right) \subseteq \tilde{\tau}_{X_{\mu}}\left(A_{\mu}, Z_{\mu}\right)+I$.

It then follows from the commutativity of the above diagram that

$$
\begin{aligned}
& \varphi_{X_{\mu}, n e_{0}}\left(F_{*}^{n e_{0}}\left(\left.\tilde{\tau}_{X_{\mu}}\left(A_{\mu}, Z_{\mu}\right)\right|_{X_{\mu}} \bar{g}^{\left(q_{0}^{n}-1\right) / r} \overline{\mathfrak{a}}_{1}^{\left\lceil t_{1}\left(q_{0}^{n}-1\right)\right\rceil} \ldots{\overline{\mathfrak{a}_{m}}}^{\left\lceil t_{m}\left(q_{0}^{n}-1\right)\right\rceil}\right)\right) \\
&\left.\subseteq \tilde{\tau}_{X_{\mu}}\left(A_{\mu}, Z_{\mu}\right)\right|_{X_{\mu}},
\end{aligned}
$$

where, for each $i=1, \ldots, m, \overline{\mathfrak{a}_{i}}$ is the image of $\mathfrak{a}_{i}$ in $\mathscr{O}_{X_{\mu}}$.

On the other hand, note that $\mathfrak{a}_{1}^{\left\lceil t_{1}\right\rceil} \ldots \mathfrak{a}_{m}^{\left\lceil t_{m}\right\rceil} \tilde{\tau}_{X_{\mu}}\left(A_{\mu}\right) \subseteq \tilde{\tau}_{X_{\mu}}\left(A_{\mu}, Z_{\mu}\right)$. By [Takagi 2010, Example 2.6], the support of $\tilde{\tau}_{X_{\mu}}\left(A_{\mu}\right)$ is contained in the singular locus of $X_{\mu}$, which does not contain any component of $D_{\mu}^{Y}$ because $X_{\mu}$ is normal. Also, by a general choice of $Y$, we may assume that no component of $D_{\mu}^{Y}$ is contained in the support of $Z_{i, \mu}$ for all $i=1, \ldots, m$. Thus, the support of $\left.\tilde{\tau}_{X_{\mu}}\left(A_{\mu}, Z_{\mu}\right)\right|_{X_{\mu}}$ 
does not contain any component of $D_{\mu}^{Y}$. By the minimality of $\tilde{\tau}_{D_{\mu}^{Y}}\left(X_{\mu},\left.Z_{\mu}\right|_{X_{\mu}}\right)$, we conclude that $\left.\tilde{\tau}_{D_{\mu}^{Y}}\left(X_{\mu},\left.Z_{\mu}\right|_{X_{\mu}}\right) \subseteq \tilde{\tau}_{X_{\mu}}\left(A_{\mu}, Z_{\mu}\right)\right|_{X_{\mu}}$.

Remark 3.3. Let the notation be as in Theorem 3.2, and fix an arbitrary point $x \in X$. Employing the same strategy as the proof of [Kawakita 2007, Theorem], we can use Theorem 3.2 to prove that the pair $\left(X ;\left.Z\right|_{X}+\frac{1}{r} V\left(J_{X}\right)\right)$ is log canonical at $x$ if and only if $(A ; c X+Z)$ is. This result is a special case of [Kawakita 2008, Theorem 1.1; Ein and Mustaţă 2009, Theorem 1.1], but our proof does not depend on the theory of jet schemes.

As a corollary, we prove the conjecture proposed in [Takagi 2010, Conjecture 2.8] when $X$ is normal and $\mathbb{Q}$-Gorenstein.

Corollary 3.4. Let A be a smooth variety over an algebraically closed field $K$ of characteristic 0 and $X \subseteq A$ be a normal $\mathbb{Q}$-Gorenstein closed subvariety of $A$. Let $Z=\sum_{i=1}^{m} t_{i} Z_{i}$ be a formal combination, where the $t_{i}$ are nonnegative real numbers and the $Z_{i} \subseteq A$ are proper closed subschemes that do not contain $X$ in their support. Given any model of $(A, X, Z)$ over a finitely generated $\mathbb{Z}$-subalgebra $B$ of $K$, there exists a dense open subset $W \subseteq \operatorname{Spec} B$ such that

$$
\operatorname{adj}_{X}(A, Z)_{\mu}=\tilde{\tau}_{X_{\mu}}\left(A_{\mu}, Z_{\mu}\right)
$$

for every closed point $\mu \in W$. In particular, the pair $(A ; Z)$ is plt along $X$ if and only if it is of purely $F$-regular type along $X$.

Proof. Let $r$ be the Gorenstein index of $X$ and $J_{X} \subseteq 0_{X}$ be the 1.c.i. defect ideal sheaf of $X$. Let $\left(A_{B}, X_{B}, Z_{B}, J_{X, B}\right)$ be any model of $\left(A, X, Z, J_{X}\right)$ over a finitely generated $\mathbb{Z}$-subalgebra $B$ of $K$. By [Takagi 2010, Theorem 2.7], there exists a dense open subset $W \subseteq \operatorname{Spec} B$ such that

$$
\tilde{\tau}_{X_{\mu}}\left(A_{\mu}, Z_{\mu}\right) \subseteq \operatorname{adj}_{X}(A, Z)_{\mu}
$$

for all closed points $\mu \in W$. Therefore, we will prove the reverse inclusion.

As an application of Theorem 2.3 to $(\star)$ and $(\star \star)$ in the proof of Theorem 3.2, after replacing $W$ by a smaller dense open subset if necessary, we may assume that

$$
\left.\operatorname{adj}_{X}(A, Z)_{\mu}\right|_{X_{\mu}}=\left.\mathscr{F}\left(X,\left.Z\right|_{X}+\frac{1}{r} V\left(J_{X}\right)\right)_{\mu} \subseteq \tilde{\tau}_{X_{\mu}}\left(A_{\mu}, Z_{\mu}\right)\right|_{X_{\mu}},
$$

that is,

$$
\operatorname{adj}_{X}(A, Z)_{\mu} \subseteq \tilde{\tau}_{X_{\mu}}\left(A_{\mu}, Z_{\mu}\right)+\Phi_{X_{\mu}}
$$

for all closed points $\mu \in W$. It, however, follows from Theorem 2.3 and [Eisenstein 2010, Theorem 5.1] that we may assume that, for all closed points $\mu \in W$,

$$
\operatorname{adj}_{X}(A, Z)_{\mu} \cap \Phi_{X_{\mu}}=\mathscr{F}(A, c X+Z)_{\mu}=\tilde{\tau}\left(A_{\mu}, c X_{\mu}+Z_{\mu}\right) \subseteq \tilde{\tau}_{X_{\mu}}\left(A_{\mu}, Z_{\mu}\right) .
$$

Thus, $\operatorname{adj}_{X}(A, Z)_{\mu} \subseteq \tilde{\tau}_{X_{\mu}}\left(A_{\mu}, Z_{\mu}\right)$ for all closed points $\mu \in W$. 


\section{The correspondence of log canonicity and F-purity when the defining equations are very general}

Using the argument developed in the previous section and involving the 1.c.i. defect ideal sheaf, we will show that Conjecture 2.4 holds true if the defining equations of the variety are very general. The following result is a generalization of a result of Hernández [2011] to the singular case:

Theorem 4.1. Let $\mathbb{A}_{K}^{n}:=\operatorname{Spec} K\left[x_{1}, \ldots, x_{n}\right]$ be the affine $n$-space over an algebraically closed field $K$ of characteristic 0 and $X \subseteq \mathbb{A}_{K}^{n}$ be a normal $\mathbb{Q}$-Gorenstein closed subvariety of codimension c passing through the origin 0. Let $r$ denote the Gorenstein index of $X$ and $J_{X}$ denote the l.c.i. defect ideal of $X$. Let $\mathfrak{a} \subseteq O_{X}$ be a nonzero ideal and $t>0$ be a real number. Suppose that there exist a system of generators $h_{1}, \ldots, h_{l}$ for the defining ideal $\mathscr{I}_{X}$ of $X$ and a system of generators $h_{l+1}, \ldots, h_{v}$ for $\mathfrak{a}$ with the following property: for each $i=1, \ldots, v$, we can write $h_{i}=\sum_{j=1}^{\rho_{i}} \gamma_{i j} x_{1}^{\alpha_{i j}^{(1)}} \ldots x_{n}^{\alpha_{i j}^{(n)}} \in K\left[x_{1}, \ldots, x_{n}\right] \quad\left(\left(\alpha_{i j}^{(1)}, \ldots, \alpha_{i j}^{(n)}\right) \in \mathbb{Z}_{\geq 0}^{n} \backslash\{\mathbf{0}\}, \gamma_{i j} \in K^{*}\right)$ with $\gamma_{i 1}, \ldots, \gamma_{i \rho_{i}}$ algebraically independent over $\mathbb{Q}$. Then $\left(X ; t V(\mathfrak{a})+\frac{1}{r} V\left(J_{X}\right)\right)$ is $\log$ canonical at 0 if and only if it is of dense sharply $F$-pure type at 0.

Remark 4.2. By the definition of $J_{X}, X$ is 1.c.i. if and only if $J_{X}=\mathrm{O}_{X}$. Thus, if $X=\operatorname{Spec} K\left[x_{1}, \ldots, x_{n}\right] /\left(h_{1}, \ldots, h_{l}\right)$ is a normal complete intersection variety, $\mathfrak{a} \subseteq$ $\mathcal{O}_{X}$ is the image of the ideal generated by $h_{l+1}, \ldots, h_{v}$ and the $h_{i} \in K\left[x_{1}, \ldots, x_{n}\right]$ satisfy the same property as that in Theorem 4.1 , then Theorem 4.1 says that $(X, t V(\mathfrak{a}))$ is $\log$ canonical at 0 if and only if it is of dense sharply $F$-pure type.

Proof. By Remark 2.5, it suffices to show that if $\left(X ; t V(\mathfrak{a})+\frac{1}{r} V\left(J_{X}\right)\right)$ is $\log$ canonical at 0 , then it is of dense sharply F-pure type.

Suppose that $\left(X ; t V(\mathfrak{a})+\frac{1}{r} V\left(J_{X}\right)\right)$ is log canonical at 0 . Since the log canonical threshold $\operatorname{lct}_{0}\left(\left(X ; \frac{1}{r} V\left(J_{X}\right)\right) ; V(\mathfrak{a})\right)$ is a rational number, we may assume that $t$ is a rational number. Take a sufficiently general complete intersection closed subscheme $Y:=V\left(\left(f_{1}, \ldots, f_{c}\right)\right)$ of codimension $c$ containing $X$, and let $s:=c-l+v$ and $f_{c+j}:=h_{l+j}$ for every $j=1, \ldots, s-c$. For each $i=1, \ldots, s$, we can write

$$
f_{i}=\sum_{j=1}^{m_{i}} u_{i j} x_{1}^{a_{i j}^{(1)}} \ldots x_{n}^{a_{i j}^{(n)}} \in K\left[x_{1}, \ldots, x_{n}\right] \quad\left(\left(a_{i j}^{(1)}, \ldots, a_{i j}^{(n)}\right) \in \mathbb{Z}_{\geq 0}^{n} \backslash\{\mathbf{0}\}, u_{i j} \in K^{*}\right),
$$

where $u_{11}, \ldots, u_{1 m_{1}}, \ldots, u_{s 1}, \ldots, u_{s m_{s}}$ are algebraically independent over $\mathbb{Q}$. We decompose $Y$ into the scheme-theoretic union of $X$ and a variety $C^{Y}$ and let $D^{Y}$ denote the Weil divisor on $X$ obtained by restricting $C^{Y}$ to $X$. Let $g \in K\left[x_{1}, \ldots, x_{n}\right]$ be a polynomial whose image is a local equation of the Cartier divisor $r D^{Y}$ in a 
neighborhood of 0 . Using the standard decent theory of [Hochster and Huneke 1999, Chapter 2], we can choose a model

$$
\left(\mathbb{A}_{B}^{n}=\operatorname{Spec} B\left[x_{1}, \ldots, x_{n}\right], X_{B}, Y_{B}=V\left(\left(f_{1, B}, \ldots, f_{c, B}\right)\right), D_{B}^{Y}, \mathfrak{a}_{B}, J_{X, B}, g_{B}\right)
$$

of $\left(\mathbb{A}_{K}^{n}, X, Y, D^{Y}, \mathfrak{a}, J_{X}, g\right)$ over a finitely generated $\mathbb{Z}$-subalgebra $B$ of $K$ satisfying these conditions, for all closed points $\mu \in \operatorname{Spec} B$ :

(i) $\mathbb{Z}\left[u_{11}, \ldots, u_{1 m_{1}}, \ldots, u_{s 1}, \ldots, u_{s m_{s}}, 1 /\left(\prod_{i, j} u_{i j}\right)\right] \subseteq B$.

(ii) The image of $g_{B}$ lies in $J_{B}$.

(iii) $X_{\mu}$ is a normal $\mathbb{Q}$-Gorenstein closed subvariety of codimension $c$ passing the origin 0 with Gorenstein index $r$.

(iv) $Y_{\mu}$ is a complete intersection closed subscheme of codimension $c$ containing $X_{\mu}$.

(v) $r D_{\mu}^{Y}$ is a Cartier divisor on $X_{\mu}$ and $\mathcal{O}_{X_{\mu}}\left(r K_{X_{\mu}}\right)=\mathcal{O}_{X_{\mu}}\left(-r D_{\mu}^{Y}\right) \omega_{Y_{\mu}}^{\otimes r}$.

(vi) The image of $g_{\mu}$ is a local equation of $r D_{\mu}^{Y}$ at 0 .

It is then enough to show that there exists a dense subset of closed points $W \subseteq \operatorname{Spec} B$ such that $\left(X_{\mu} ; t V\left(\mathfrak{a}_{\mu}\right)+\frac{1}{r} V\left(J_{X, \mu}\right)\right)$ is sharply $F$-pure at 0 for all $\mu \in W$.

Since $\left(X ; t V(\mathfrak{a})+\frac{1}{r} V\left(J_{X}\right)\right)$ is $\log$ canonical at 0 , it follows from [Kawakita 2008, Theorem 1.1; Ein and Mustaţă 2009, Theorem 1.1] (see also Remark 3.3) that $\left(\mathbb{A}_{K}^{n} ; t V(\mathfrak{a})+c X\right)$ is $\log$ canonical at 0 . By a general choice of $f_{1}, \ldots, f_{c}$, it is equivalent to saying that

$$
\left(\mathbb{A}_{K}^{n} ; \sum_{i=1}^{c} \operatorname{div}\left(f_{i}\right)+t V\left(f_{c+1}, \ldots, f_{s}\right)\right)
$$

is $\log$ canonical at 0 . By making use of the summation formula for multiplier ideals [Takagi 2006, Theorem 3.2], for any $\epsilon>0$, there exist nonnegative rational numbers $\lambda_{c+1}(\epsilon), \ldots, \lambda_{s}(\epsilon)$ with $\lambda_{c+1}(\epsilon)+\cdots+\lambda_{s}(\epsilon)=t(1-\epsilon)$ such that

$$
\left(\mathbb{A}_{K}^{n} ; \sum_{i=1}^{c}(1-\epsilon) \operatorname{div}\left(f_{i}\right)+\sum_{j=c+1}^{s} \lambda_{j}(\epsilon) \operatorname{div}\left(f_{j}\right)\right)
$$

is klt at 0 . Let $\mathfrak{a}_{f_{i}}$ be the term ideal of $f_{i}$ (that is, the monomial ideal generated by the terms of $f_{i}$ ) for each $i=1, \ldots, s$. Since $\mathfrak{a}_{f_{i}}$ contains $f_{i}$, the monomial ideal $\mathscr{g}\left(\mathbb{A}_{K}^{n}, \sum_{i=1}^{c}(1-\epsilon) V\left(\mathfrak{a}_{f_{i}}\right)+\sum_{j=c+1}^{s} \lambda_{j}(\epsilon) V\left(\mathfrak{a}_{f_{j}}\right)\right)$ is trivial. Then by the Main Theorem of [Howald 2001], the vector 1 lies in the interior of

$$
\sum_{i=1}^{c}(1-\epsilon) P\left(\mathfrak{a}_{f_{i}}\right)+\sum_{j=c+1}^{s} \lambda_{j}(\epsilon) P\left(\mathfrak{a}_{f_{j}}\right),
$$


where $P\left(\mathfrak{a}_{f_{i}}\right)$ is the Newton polyhedron of $\mathfrak{a}_{f_{i}}$ for each $i=1, \ldots, s$. This is equivalent to saying that there exists

$$
\sigma(\epsilon)=\left(\sigma_{11}(\epsilon), \ldots, \sigma_{1 m_{1}}(\epsilon), \ldots, \sigma_{s 1}(\epsilon), \ldots, \sigma_{s m_{s}}(\epsilon)\right) \in \mathbb{R}_{\geq 0}^{\sum_{i=1}^{s} m_{i}}
$$

such that

(1) $A \sigma(\epsilon)^{\mathrm{T}}<\mathbf{1}$

(2) $\sum_{j=1}^{m_{i}} \sigma_{i j}(\epsilon)=1-\epsilon$ for every $i=1, \ldots, c$, and

(3) $\sum_{j=1}^{m_{i}} \sigma_{i j}(\epsilon)=\lambda_{i}(\epsilon)$ for every $i=c+1, \ldots, s$,

where $A$ is the $n \times \sum_{i=1}^{s} m_{i}$ matrix

$$
\left(\begin{array}{cccccccc}
a_{11}^{(1)} & \ldots & a_{1 m_{1}}^{(1)} & a_{21}^{(1)} & \ldots & a_{s 1}^{(1)} & \ldots & a_{s m_{s}}^{(1)} \\
\vdots & \ddots & \vdots & \vdots & & \vdots & \ddots & \vdots \\
a_{11}^{(n)} & \ldots & a_{1 m_{1}}^{(n)} & a_{21}^{(n)} & \ldots & a_{s 1}^{(n)} & \ldots & a_{s m_{s}}^{(n)}
\end{array}\right) .
$$

Since such a $\sigma(\epsilon)$ exists for every $\epsilon>0$, by the continuity of real numbers and the convexity of the solution space

$$
\left\{\boldsymbol{\tau} \in \mathbb{R}_{\geq 0}^{\sum_{i=1}^{s} m_{i}} \mid \tilde{A} \boldsymbol{\tau}^{\mathrm{T}} \leq \mathbf{1}\right\}
$$

there exists $\sigma=\left(\sigma_{11}, \ldots, \sigma_{1 m_{1}}, \ldots, \sigma_{s 1}, \ldots, \sigma_{s m_{s}}\right) \in \mathbb{Q}_{\geq 0}^{\sum_{i=1}^{s} m_{i}}$ such that

(更) $\widetilde{A} \sigma^{\mathrm{T}} \leq \mathbf{1}$,

(2) $\sum_{j=1}^{m_{i}} \sigma_{i j}=1$ for every $i=1, \ldots, c$, and

(苂) $\sum_{i=c+1}^{s} \sum_{j=1}^{m_{i}} \sigma_{i j}=t$,

where $\widetilde{A}$ is the $(n+s) \times \sum_{i=1}^{s} m_{i}$ matrix

$$
\left(\begin{array}{ccccccccccc}
a_{11}^{(1)} & \ldots & a_{1 m_{1}}^{(1)} & a_{21}^{(1)} & \ldots & a_{2 m_{2}}^{(1)} & a_{31}^{(1)} & \ldots & a_{s 1}^{(1)} & \ldots & a_{s m_{s}}^{(1)} \\
\vdots & \ddots & \vdots & \vdots & \ddots & \vdots & \vdots & & \vdots & \ddots & \vdots \\
a_{11}^{(n)} & \ldots & a_{1 m_{1}}^{(n)} & a_{21}^{(n)} & \ldots & a_{2 m_{2}}^{(n)} & a_{31}^{(n)} & \ldots & a_{s 1}^{(n)} & \ldots & a_{s m_{s}}^{(n)} \\
1 & \ldots & 1 & 0 & \ldots & 0 & 0 & \ldots & 0 & \ldots & 0 \\
& & & 1 & \ldots & 1 & 0 & \ldots & 0 & \ldots & 0 \\
& & & & & & 1 & \ldots & 0 & \ldots & 0 \\
& & & & & & & & \vdots & & \vdots \\
& & & & & & & & 0 & \ldots & 0 \\
& & & & & & & & 1 & \ldots & 1
\end{array}\right) .
$$

We take the least common multiple $N$ of the denominators of the $\sigma_{i j}$ so that $\sigma_{i j}(p-1)$ is an integer for all $i=1, \ldots, s$ and all $j=1, \ldots, m_{i}$ whenever $p \equiv 1 \bmod N$.

Let $p$ be a prime such that $p \equiv 1 \bmod N r$, and let $e_{1}, \ldots, e_{n}$ be nonnegative 
integers such that

$$
(p-1) \tilde{A} \sigma^{\mathrm{T}}=\left(e_{1}, \cdots, e_{n}, \sum_{j=1}^{m_{1}} \sigma_{1 j}(p-1), \cdots, \sum_{j=1}^{m_{s}} \sigma_{s j}(p-1)\right)^{\mathrm{T}} .
$$

Then $e_{k} \leq p-1$ for all $k=1, \ldots, n$. The coefficient of the monomial $x_{1}^{e_{1}} \cdots x_{n}^{e_{n}}$ in the expansion of

$$
f_{1}^{\sum_{j=1}^{m_{1}} \sigma_{1 j}(p-1)} \cdots f_{s}^{\sum_{j=1}^{m_{s}} \sigma_{s j}(p-1)}
$$

is

$$
\theta_{\boldsymbol{\sigma}, p}(\boldsymbol{u}):=\sum_{\tau_{i j}} \prod_{i=1}^{s}\left(\begin{array}{c}
\sum_{j=1}^{m_{i}} \sigma_{i j}(p-1) \\
\tau_{i 1}, \ldots, \tau_{i m_{i}}
\end{array}\right) u_{i 1}^{\tau_{i 1}} \cdots u_{i m_{i}}^{\tau_{i m_{i}}} \in \mathbb{Z}\left[u_{i j}\right] \underset{\substack{i=1, \ldots, s \\
j=1, \ldots, m_{i}}}{\subseteq} \subseteq B,
$$

where the summation runs over all $\tau=\left(\tau_{11}, \ldots, \tau_{1 m_{1}}, \ldots, \tau_{s 1}, \ldots, \tau_{s m_{s}}\right) \in \mathbb{Z}_{\geq 0}^{\sum_{i=1}^{s} m_{i}}$ such that

$$
\widetilde{A} \tau^{\mathrm{T}}=\left(e_{1}, \cdots, e_{n}, \sum_{j=1}^{m_{1}} \sigma_{1 j}(p-1), \cdots, \sum_{j=1}^{m_{s}} \sigma_{s j}(p-1)\right)^{\mathrm{T}} .
$$

Since $\widetilde{A} \sigma^{\mathrm{T}} \leq \mathbf{1}$, one has $\sum_{j=1}^{m_{i}} \sigma_{i j}(p-1) \leq p-1$ for all $i=1, \ldots, s$, so the coefficient

$$
\prod_{i=1}^{s}\left(\begin{array}{c}
\sum_{j=1}^{m_{i}} \sigma_{i j}(p-1) \\
\sigma_{i 1}(p-1), \ldots, \sigma_{i m_{i}}(p-1)
\end{array}\right)
$$

of the monomial $\prod_{i=1}^{s} u_{i 1}^{\sigma_{i 1}(p-1)} \cdots u_{i m_{i}}^{\sigma_{i m_{i}}(p-1)}$ in $\theta_{\sigma, p}(\boldsymbol{u})$ is nonzero in $\mathbb{F}_{p}$. This means that $\theta_{\sigma, p}(\boldsymbol{u})$ is nonzero in $\mathbb{F}_{p}\left[u_{i j}\right]_{i=1, \ldots, s, j=1, \ldots, m_{i}} \subseteq B / p B$ because, by assumption, the $u_{i j}$ are algebraically independent over $\mathbb{F}_{p}$, so $D\left(\theta_{\sigma, p}(\boldsymbol{u})\right) \cap \operatorname{Spec} B / p B$ is a dense open subset of $\operatorname{Spec} B / p B$.

We now set

$$
W:=\bigcup_{p \equiv 1 \bmod N r} D\left(\theta_{\sigma, p}(\boldsymbol{u})\right) \cap \operatorname{Spec} B / p B \subseteq \operatorname{Spec} B .
$$

Then $W$ is a dense subset of $\operatorname{Spec} B$. Fix any closed point $\mu \in W$, and let $p$ denote the characteristic of the residue field $\kappa(\mu)=B / \mu$ from now on. Since the image of $\theta_{\sigma, p}(\boldsymbol{u})$ is nonzero in $B / \mu$, the monomial $x_{1}^{e_{1}} \cdots x_{n}^{e_{n}}$ appears in the expansion of

$$
f_{1, \mu}^{\left(\sum_{j=1}^{m_{1}} \sigma_{1 j}\right)(p-1)} \cdots f_{s, \mu}^{\left(\sum_{j=1}^{m_{s}} \sigma_{s j}\right)(p-1)}
$$

in $(B / \mu)\left[x_{1}, \ldots, x_{n}\right]$. Since $\sum_{j=1}^{m_{i}} \sigma_{i j}(p-1)=p-1$ for all $i=1, \ldots, c$ and $e_{k} \leq p-1$ for all $k=1, \ldots, n$, one has

$$
f_{1, \mu}^{p-1} \cdots f_{c, \mu}^{p-1} f_{c+1, \mu}^{\left(\sum_{j=1}^{m_{c+1}} \sigma_{c+1 j}\right)(p-1)} \cdots f_{s, \mu}^{\left(\sum_{j=1}^{m_{s}} \sigma_{s j}\right)(p-1)} \notin\left(x_{1}^{p}, \ldots, x_{n}^{p}\right)
$$


in $(B / \mu)\left[x_{1}, \ldots, x_{n}\right]_{\left(x_{1}, \ldots, x_{n}\right)}$. By Lemma $1.5(2)$, this is equivalent to saying that for all powers $q=p^{e}$ of $p$,

$$
f_{1, \mu}^{q-1} \cdots f_{c, \mu}^{q-1} f_{c+1, \mu}^{\left(\sum_{j=1}^{m_{c+1}} \sigma_{c+1 j}\right)(q-1)} \cdots f_{s, \mu}^{\left(\sum_{j=1}^{m_{s}} \sigma_{s j}\right)(q-1)} \notin\left(x_{1}^{q}, \ldots, x_{n}^{q}\right)
$$

in $(B / \mu)\left[x_{1}, \ldots, x_{n}\right]_{\left(x_{1}, \ldots, x_{n}\right)}$. Applying the claim in the proof of Theorem 3.2, one has

$$
\left(\Phi_{X, \mu}^{[q]}: \Phi_{X, \mu}\right) g_{\mu}^{(q-1) / r} f_{c+1, \mu}^{\left(\sum_{j=1}^{m_{c+1}} \sigma_{c+1 j}\right)(q-1)} \cdots f_{s, \mu}^{\left(\sum_{j=1}^{m_{s}} \sigma_{s j}\right)(q-1)} \notin\left(x_{1}^{q}, \ldots, x_{n}^{q}\right)
$$

in $(B / \mu)\left[x_{1}, \ldots, x_{n}\right]_{\left(x_{1}, \ldots, x_{n}\right)}$. Since $\sum_{i=c+1}^{s} \sum_{j=1}^{m_{i}} \sigma_{i j}=t$ and the image of $g_{\mu}$ lies in $J_{X, \mu}$, it follows from Lemma 1.5(2) again that the pair $\left(X_{\mu} ; \frac{1}{r} V\left(J_{X, \mu}\right)+t V\left(\mathfrak{a}_{\mu}\right)\right)$ is sharply $F$-pure at 0 .

Remark 4.3. Using the same arguments as the proof of Theorem 4.1, we can prove the following. Let $X=\operatorname{Spec} K\left[x_{1}, \ldots, x_{n}\right] /\left(f_{1}, \ldots, f_{c}\right)$ be a normal complete intersection over a field $K$ of characteristic 0 passing through the origin 0 . Let $Z \subset X$ be a proper closed subscheme passing through 0 and $f_{c+1}, \ldots, f_{s}$ be a system of polynomials whose image generates the defining ideal $\Phi_{Z} \subseteq O_{X}$ of $Z$. We write

$$
f_{i}=\sum_{j=1}^{m_{i}} u_{i j} x_{1}^{a_{i j}^{(1)}} \cdots x_{n}^{a_{i j}^{(n)}} \in K\left[x_{1}, \ldots, x_{n}\right] \quad\left(\left(a_{i j}^{(1)}, \ldots, a_{i j}^{(n)}\right) \in \mathbb{Z}_{\geq 0}^{n} \backslash\{\mathbf{0}\}, u_{i j} \in K^{*}\right)
$$

for each $i=1, \ldots, s$ and set $A$ to be the $(n+s) \times \sum_{i=1}^{s} m_{i}$ matrix from $(\triangle)$. Then we consider the following linear programming problem:

$$
\begin{aligned}
\text { Maximize } & \sum_{i=c+1}^{s} \sum_{j=1}^{m_{i}} \sigma_{i j} \\
\text { subject to } & A\left(\sigma_{11}, \ldots, \sigma_{1 m_{1}}, \ldots, \sigma_{s 1}, \ldots, \sigma_{s m_{s}}\right)^{\mathrm{T}} \leq \mathbf{1}, \\
& \sum_{i=1}^{c} \sum_{j=1}^{m_{i}} \sigma_{i j}=c, \\
& \sigma_{i j} \in \mathbb{Q}_{\geq 0} \text { for all } i=1, \ldots, s \text { and all } j=1, \ldots, m_{i} .
\end{aligned}
$$

Assume that there exists an optimal solution $\sigma=\left(\sigma_{11}, \ldots, \sigma_{1 m_{1}}, \ldots, \sigma_{s 1}, \ldots, \sigma_{s m_{s}}\right)$ such that $A \sigma^{\mathrm{T}} \neq A \boldsymbol{\sigma}^{\prime \mathrm{T}}$ for all other optimal solutions $\sigma^{\prime} \neq \sigma$. In addition, we assume that $X$ is $\log$ canonical at 0 . Then:

(1) $\operatorname{lct}_{0}(X, Z)$ is equal to the optimal value $\sum_{i=c+1}^{s} \sum_{j=1}^{m_{i}} \sigma_{i j}$.

(2) Given any model of $(X, Z)$ over a finitely generated $\mathbb{Z}$-subalgebra $B$ of $K$, there exists a dense subset of closed points $W \subseteq \operatorname{Spec} B$ such that

$$
\operatorname{lct}_{0}(X ; Z)=\operatorname{fpt}_{0}\left(X_{\mu} ; Z_{\mu}\right) \quad \text { for all } \mu \in W .
$$


Shibuta and Takagi [2009] showed that the assumption of Remark 4.3 is satisfied if $X=\mathbb{A}_{K}^{n}$ and $Z$ is a complete intersection binomial subscheme or a space monomial curve (in the latter case, $n=3$ ). However, in general, there exists a binomial subscheme that does not satisfy the assumption.

Example 4.4. Let $X:=\mathbb{A}_{K}^{6}=\operatorname{Spec} K\left[x_{1}, x_{2}, x_{3}, y_{1}, y_{2}, y_{3}\right]$ be the affine 6-space over a field $K$ of characteristic 0 and $Z \subseteq X$ be the closed subscheme defined by the binomials $x_{1} y_{2}-x_{2} y_{1}, x_{2} y_{3}-x_{3} y_{2}$ and $x_{1} y_{3}-x_{3} y_{1}$. Then $Z$ does not satisfy the assumption of Remark 4.3. Indeed, $\operatorname{lct}_{0}(X, Z)=2$, but the optimal value of the linear programming problem in Remark 4.3 is equal to 3 . Given a prime number $p$, let $X_{p}:=\mathbb{A}_{\mathbb{F}_{p}}^{6}=\operatorname{Spec} \mathbb{F}_{p}\left[x_{1}, x_{2}, x_{3}, y_{1}, y_{2}, y_{3}\right]$ and $Z_{p} \subseteq X_{p}$ be the reduction modulo $p$ of $Z$. Since $\operatorname{fpt}_{0}\left(X_{p}, Z_{p}\right)=2$ for all primes $p$, Conjecture 2.4 holds for this example.

\section{Acknowledgments}

The author is grateful to Daniel Hernández, Nobuo Hara, Junmyeong Jang, Masayuki Kawakita, Karl Schwede and Takafumi Shibuta for valuable conversations. He is indebted to Natsuo Saito for his help with $\mathrm{LT}_{\mathrm{E}} \mathrm{X}$. He also would like to thank Shihoko Ishii and an anonymous referee for pointing out mistakes in a previous version of this paper. The author would like to express his gratitude to the Massachusetts Institute of Technology, where a part of this work was done, for their hospitality during the winter of 2010-2011. The author was partially supported by Grant-in-Aid for Young Scientists (B) 20740019 and 23740024 from JSPS and by Program for Improvement of Research Environment for Young Researchers from SCF commissioned by MEXT of Japan.

\section{References}

[Blickle 2004] M. Blickle, "Multiplier ideals and modules on toric varieties", Math. Z. 248:1 (2004), 113-121. MR 2006a:14082 Zbl 1061.14055

[Ein and Mustață 2009] L. Ein and M. Mustaţă, "Jet schemes and singularities", pp. 505-546 in Algebraic geometry. Part 2 (Seattle, 2005), edited by D. Abramovich et al., Proc. Sympos. Pure Math. 80, Amer. Math. Soc., Providence, RI, 2009. MR 2010h:14004 Zbl 1181.14019

[Eisenstein 2010] E. Eisenstein, "Generalization of the restriction theorem for multiplier ideals", preprint, 2010. arXiv 1001.2841

[Fedder 1983] R. Fedder, “F-purity and rational singularity”, Trans. Amer. Math. Soc. 278:2 (1983), 461-480. MR 84h:13031 Zbl 0519.13017

[Hara 1998] N. Hara, "A characterization of rational singularities in terms of injectivity of Frobenius maps", Amer. J. Math. 120:5 (1998), 981-996. MR 99h:13005 Zbl 0942.13006

[Hara and Watanabe 2002] N. Hara and K.-I. Watanabe, "F-regular and F-pure rings vs. log terminal and log canonical singularities", J. Algebraic Geom. 11:2 (2002), 363-392. MR 2002k:13009 Zbl 1013.13004 
[Hara and Yoshida 2003] N. Hara and K.-I. Yoshida, "A generalization of tight closure and multiplier ideals”, Trans. Amer. Math. Soc. 355:8 (2003), 3143-3174. MR 2004i:13003 Zbl 1028.13003

[Hernández 2011] D. Hernández, “F-purity versus log canonicity for polynomials”, preprint, 2011. arXiv 1112.2423

[Hironaka 1964] H. Hironaka, "Resolution of singularities of an algebraic variety over a field of characteristic zero. I, II”, Ann. of Math. 79 (1964), 205-326. MR 33 \#7333 Zbl 0122.38603

[Hochster and Huneke 1999] M. Hochster and C. Huneke, "Tight closure in equal characteristic zero", preprint, 1999, http://www.math.lsa.umich.edu/ hochster/tcz.ps.

[Howald 2001] J. A. Howald, "Multiplier ideals of monomial ideals", Trans. Amer. Math. Soc. 353:7 (2001), 2665-2671. MR 2002b:14061 Zbl 0979.13026

[Jang 2011] J. Jang, "The ordinarity of an isotrivial elliptic fibration", Manuscripta Math. 134:3-4 (2011), 343-358. MR 2012f:14014 Zbl 1225.14027

[Kawakita 2007] M. Kawakita, "Inversion of adjunction on log canonicity", Invent. Math. 167:1 (2007), 129-133. MR 2008a:14025 Zbl 1114.14009

[Kawakita 2008] M. Kawakita, "On a comparison of minimal log discrepancies in terms of motivic integration”, J. Reine Angew. Math. 620 (2008), 55-65. MR 2010i:14021 Zbl 1151.14014

[Kawamata 1988] Y. Kawamata, "Crepant blowing-up of 3-dimensional canonical singularities and its application to degenerations of surfaces”, Ann. of Math. (2) 127:1 (1988), 93-163. MR 89d:14023 Zbl 0651.14005

[Kollár and Mori 1998] J. Kollár and S. Mori, Birational geometry of algebraic varieties, Cambridge Tracts in Mathematics 134, Cambridge University Press, 1998. MR 2000b:14018 Zbl 0926.14003

[Lazarsfeld 2004] R. Lazarsfeld, Positivity in algebraic geometry. II, Ergebnisse der Mathematik und ihrer Grenzgebiete. 3. Folge. A Series of Modern Surveys in Mathematics 49, Springer, Berlin, 2004. MR 2005k:14001b Zbl 1093.14500

[Mehta and Srinivas 1991] V. B. Mehta and V. Srinivas, "Normal F-pure surface singularities", $J$. Algebra 143:1 (1991), 130-143. MR 92j:14044 Zbl 0760.14012

[Miller and Schwede 2012] L. E. Miller and K. Schwede, "Semi-log canonical vs $F$-pure singularities”, J. Algebra 349 (2012), 150-164. MR 2853631 Zbl 1239.13010

[Mustaţă and Srinivas 2011] M. Mustață and V. Srinivas, "Ordinary varieties and the comparison between multiplier ideals and test ideals", Nagoya Math. J. 204 (2011), 125-157. MR 2863367 Zbl 1239.14011

[Ogus 1982] A. Ogus, "Hodge cycles and crystalline cohomology", pp. 357-414 in Hodge cycles, motives, and Shimura varieties, edited by P. Deligne et al., Lecture Notes in Mathematics 900, Springer, Berlin, 1982. MR 84m:14046 Zbl 0538.14010

[Schwede 2008] K. Schwede, "Generalized test ideals, sharp F-purity, and sharp test elements", Math. Res. Lett. 15:6 (2008), 1251-1261. MR 2010e:13004 Zbl 1185.13010

[Schwede 2009] K. Schwede, “F-adjunction”, Algebra Number Theory 3:8 (2009), 907-950. MR 2011b:14006 Zbl 1209.13013

[Schwede 2011] K. Schwede, “Test ideals in non- $\mathbb{Q}-G o r e n s t e i n$ rings”, Trans. Amer. Math. Soc. 363:11 (2011), 5925-5941. MR 2012c:13011 Zbl 05986706

[Schwede and Tucker 2012] K. Schwede and K. Tucker, "On the behavior of test ideals under finite morphisms", preprint, 2012. To appear in J. Algebraic Geom. arXiv 1003.4333

[Serre 1966] J.-P. Serre, “Groupes de Lie $l$-adiques attachés aux courbes elliptiques”, pp. 239-256 in Les tendances géométriques en algébre et théorie des nombres, Centre National de la Recherche Scientifique, Paris, 1966. MR 36 \#1453 Zbl 0148.41502 
[Shibuta and Takagi 2009] T. Shibuta and S. Takagi, "Log canonical thresholds of binomial ideals", Manuscripta Math. 130:1 (2009), 45-61. MR 2010j:14031 Zbl 1183.13007

[Takagi 2004a] S. Takagi, "F-singularities of pairs and inversion of adjunction of arbitrary codimension", Invent. Math. 157:1 (2004), 123-146. MR 2006g:14028 Zbl 1121.13008

[Takagi 2004b] S. Takagi, "An interpretation of multiplier ideals via tight closure", J. Algebraic Geom. 13:2 (2004), 393-415. MR 2005c:13002 Zbl 1080.14004

[Takagi 2006] S. Takagi, "Formulas for multiplier ideals on singular varieties", Amer. J. Math. 128:6 (2006), 1345-1362. MR 2007i:14006 Zbl 1109.14005

[Takagi 2008] S. Takagi, "A characteristic $p$ analogue of plt singularities and adjoint ideals", Math. Z. 259:2 (2008), 321-341. MR 2009b:13004 Zbl 1143.13007

[Takagi 2010] S. Takagi, "Adjoint ideals along closed subvarieties of higher codimension", J. Reine Angew. Math. 641 (2010), 145-162. MR 2011f:14032 Zbl 1193.14024

[Watanabe 1988] K. Watanabe, "Study of F-purity in dimension two", pp. 791-800 in Algebraic geometry and commutative algebra, vol. II, edited by H. Hijikata et al., Kinokuniya, Tokyo, 1988. MR 90b:14005 Zbl 0780.13004

Communicated by Craig Huneke

Received 2011-07-01 Revised 2012-04-23 Accepted 2012-05-27

stakagi@ms.u-tokyo.ac.jp Graduate School of Mathematical Sciences, University of Tokyo, 3-8-1 Komaba, Meguro-ku, Tokyo 153-8914, Japan http://www.ms.u-tokyo.ac.jp/ stakagi/ 


\section{Algebra \& Number Theory}

msp.org/ant

\section{EDITORS}

MANAGING EDITOR

Bjorn Poonen

Massachusetts Institute of Technology

Cambridge, USA

\author{
EDITORIAL BOARD CHAIR \\ David Eisenbud \\ University of California \\ Berkeley, USA
}

\section{BOARD OF EDITORS}

Georgia Benkart

Dave Benson

Richard E. Borcherds

John H. Coates

J-L. Colliot-Thélène

Brian D. Conrad

Hélène Esnault

Hubert Flenner

Edward Frenkel

Andrew Granville

Joseph Gubeladze

Ehud Hrushovski

Craig Huneke

Mikhail Kapranov

Yujiro Kawamata

János Kollár

Yuri Manin

Barry Mazur

Philippe Michel
University of Wisconsin, Madison, USA

University of Aberdeen, Scotland

University of California, Berkeley, USA

University of Cambridge, UK

CNRS, Université Paris-Sud, France

University of Michigan, USA

Freie Universität Berlin, Germany

Ruhr-Universität, Germany

University of California, Berkeley, USA

Université de Montréal, Canada

San Francisco State University, USA

Hebrew University, Israel

University of Virginia, USA

Yale University, USA

University of Tokyo, Japan

Princeton University, USA

Northwestern University, USA

Harvard University, USA

École Polytechnique Fédérale de Lausanne
Susan Montgomery

Shigefumi Mori

Raman Parimala

Jonathan Pila

Victor Reiner

Karl Rubin

Peter Sarnak

Joseph H. Silverman

Michael Singer

Vasudevan Srinivas

J. Toby Stafford

Bernd Sturmfels

Richard Taylor

Ravi Vakil

Michel van den Bergh

Marie-France Vignéras

Kei-Ichi Watanabe

Efim Zelmanov
University of Southern California, USA

RIMS, Kyoto University, Japan

Emory University, USA

University of Oxford, UK

University of Minnesota, USA

University of California, Irvine, USA

Princeton University, USA

Brown University, USA

North Carolina State University, USA

Tata Inst. of Fund. Research, India

University of Michigan, USA

University of California, Berkeley, USA

Harvard University, USA

Stanford University, USA

Hasselt University, Belgium

Université Paris VII, France

Nihon University, Japan

University of California, San Diego, USA

\section{PRODUCTION}

production@msp.org

Silvio Levy, Scientific Editor

See inside back cover or msp.org/ant for submission instructions.

The subscription price for 2013 is US \$200/year for the electronic version, and \$350/year ( $\$ 40$, if shipping outside the US) for print and electronic. Subscriptions, requests for back issues and changes of subscribers address should be sent to MSP.

Algebra \& Number Theory (ISSN 1944-7833 electronic, 1937-0652 printed) at Mathematical Sciences Publishers, 798 Evans Hall \#3840, c/o University of California, Berkeley, CA 94720-3840 is published continuously online. Periodical rate postage paid at Berkeley, CA 94704, and additional mailing offices.

ANT peer review and production are managed by EditFLOW ${ }^{\circledR}$ from Mathematical Sciences Publishers.

\section{PUBLISHED BY}

- mathematical sciences publishers

nonprofit scientific publishing

http://msp.org/

(C) 2013 Mathematical Sciences Publishers 


\section{Algebra \& Number Theory}

$\begin{array}{lll}\text { Volume } 7 & \text { No. } 4 \quad 2013\end{array}$

Explicit Chabauty over number fields

SAMIR SIKSEK

Moduli spaces for point modules on naïve blowups

Thomas A. Nevins and Susan J. Sierra

Density of rational points on certain surfaces

Sir Peter SWInNerton-Dyer

HENRIK RUSSELL

Chai's conjecture and Fubini properties of dimensional motivic integration

RAF Cluckers, François LoESER and JohanNes NiCAISE

SHUNSUKE TAKAGI

Finitely presented exponential fields

JONATHAN KIRBY

On a problem of Arnold: The average multiplicative order of a given integer

PÄr Kurlberg and CARL POMERANCE

An analogue of Sturm's theorem for Hilbert modular forms 